\title{
Bird diversity and conservation in the southern coast of Santa Catarina state, Brazil
}

\author{
João Paulo Gava Just'; Jonas Rafael Rodrigues Rosoni²; Rafael Spilere Romagna ${ }^{3,4}$ \& Jairo José Zocche ${ }^{3,5}$ \\ 1 Universidade Federal de Pelotas (UFPEL), Instituto de Biologia (IB), Departamento de Ecologia, Zoologia e Genética (DEZG), Programa de \\ Pós-Graduação em Biologia Animal (PPGBA). Capão do Leão, RS, Brasil. ORCID: 0000-0002-9453-4622. E-mail: joaopaulogavajust@gmail.com \\ ${ }^{2}$ Universidade Federal de Santa Maria (UFSM), Centro de Ciências Naturais e Exatas (CCNE), Programa de Pós-Graduação em Biodiversidade \\ Animal (PPGBA). Santa Maria, RS, Brasil. ORCID: 0000-0001-7556-9021. E-mail: jonas.rosoni@gmail.com \\ 3 Universidade do Extremo Sul Catarinense (UNESC), Laboratório de Ecologia de Paisagem e de Vertebrados (LABECO), \\ Programa de Pós-Graduação em Ciências Ambientais (PPGCA). Criciúma, SC, Brasil. \\ 4 ORCID: 0000-0002-6387-5985. E-mail: rafaelsromagna@outlook.com \\ 5 ORCID: 0000-0003-2291-3065. E-mail: jjz@unesc.net
}

\begin{abstract}
Coastal lagoons and their surrounding habitats often harbour high biodiversity and some of the most threatened ecosystems in the world. However, in the Neotropics the biodiversity is often poorly described, lacking even inventories of species which therefore limits the assessment of threats and the stablishment of efficient conservation measures. We present here lists of bird species recorded at ten sites along the under-studied coastal lagoons of southern Santa Catarina, Brazil, collected mainly from October 2012 to March 2018. We present quantitative data for endemic and threatened species, identify migratory status and highlight distributional novelties. In total, we recorded 229 species encompassing 63 families, including twelve species of conservation concern, 11 endemic to the Atlantic Forest or Pampas zoogeographical provinces and 38 migrants. We provide new records of seven rare species in Santa Catarina (Larus atlanticus, Calidris pusilla, Aramides ypecaha, Bubo virginianus, Limnornis curvirostris, Phacellodomus ferrugineigula and Pseudocolopteryx flaviventris) and report the first evidence of occurrence of Schoeniophylax phryganophilus for the State. Based on our findings, we discuss the regional diversity and conservation of the avifauna.
\end{abstract}

Key-Words. Species list; Coastal lagoon; Inventory; Species richness; Threatened species.

\section{INTRODUCTION}

Coastal lagoons and their surrounding environments frequently form complex mosaics of aquatic and terrestrial ecossystems that harbor high biodiversity (Esteves et al., 2008). The high productivity of such wetlands provides essencial ecosystemic services to humankind such as water supply and fishing resources (Esteves et al., 2008). One of the largest complex of lagoons in the Neotropical region is found in the southern coast of Brazil, ranging from southern Santa Catarina state to southern Rio Grande do Sul state (Burger, 1999). This landscape was created by sea movements within the last 7,000 years and currently includes lagoons associated with a diverse range of habitats as estuaries, mangroves, grasslands, wetlands, restingas and lowland forests (Burger, 1999; Tomazelli \& Villwock, 2005). These ecosystems have been intensively modified in southern Brazil due to human occupation, agriculture, pollution, and introduction of exotic species (Esteves et al., 2008).

Along with many other types of wetlands, lagoons are amongst the most threatened
Neotropical ecosystems and are considered as "extreme priority" for conservation and further research (Burger, 1999; Guadagnin \& Laidner, 1999; MMA, 2002, 2007; Burger \& Ramos, 2007; Esteves et al., 2008). Indeed, this region plays a crucial role for bird conservation as it encompasses one of the highest concentrations of aquatic birds in Brazil and is wintering ground for a number of Nearctic and Neotropical migratory species (Schott \& Carbonell, 1986; Bencke et al., 2006; Valente et al., 2011). Despite this importance, most bird inventories or related studies are from the southern portion of these wetlands (i.e., Rio Grande do Sul state: Belton, 1994; Nascimento, 1995; Mähler-Jr. et al., 1996; Guadagnin et al., 2005; Bencke et al., 2007; Guadagnin \& Maltchik, 2007; Guadagnin et al., 2009; Harrison et al., 2013; Vizentin-Bugoni et al., 2015; Dias et al., 2016), while the northern portion is poorly sampled (i.e., southern Santa Catarina state: Rosário-Bege \& Marterer, 1991; Guadagnin \& Laidner, 1999).

Ornithological surveys on the coastal lagoons of southern Santa Catarina were pioneered by the French naturalist August de Saint-Hilaire in 1820, who made relevant contributions such as 
Table 1. Study sites in the southern coast of Santa Catarina state, Brazil, with details on coordinates, size, sample effort, periodicity and habitats sampled (be $=$ beach, $\mathrm{cf}=$ cultivated field, ex = plantation of exotic trees - eucalyptus or pinus, $\mathrm{fo}=$ lowland forest, la = coastal lagoon, ma = marsh with stands of emergent macrophytes, $\mathrm{ng}=$ native grassland, $\mathrm{om}=$ open marsh, $\mathrm{pa}=$ pasture, $\mathrm{sr}=$ shrubby restinga, $\mathrm{ua}=$ urban area).

\begin{tabular}{|c|c|c|c|c|c|}
\hline Locality, Municipality & Central coordinates & Surveyed area & Effort & Period & Habitat \\
\hline 1. Lagoa da Urussanga Velha, Balneário Rincão & $28^{\circ} 46^{\prime} 45^{\prime \prime S}, 49^{\circ} 12^{\prime} 39^{\prime \prime} \mathrm{W}$ & $0.80 \mathrm{~km}^{2}$ & $28 \mathrm{~h}$ & $\begin{array}{l}\text { Jun. and Jul. 2013, Dec. 2015, Jan. 2016, } \\
\text { Nov. 2016, Jan. } 2017\end{array}$ & ar, fo, la, ma, ng, om, pa \\
\hline 2. Praia do Rincão, Balneário Rincão & $28^{\circ} 52^{\prime} 00^{\prime \prime} S, 49^{\circ} 16^{\prime} 20^{\prime \prime} \mathrm{W}$ & $4.75 \mathrm{~km}^{2}$ & $70 \mathrm{~h}$ & $\begin{array}{l}\text { Oct. 2012, Feb. } 2014 \text { to Jan. 2015, Dec. 2015, } \\
\text { Jan. 2016, Ago. 2016, Jan. and Mar. } 2018\end{array}$ & ar, be, cf, ex, la, ma, ng, om, pa, ua \\
\hline 3. Lagoa do Rincão/Jacaré, Balneário Rincão & $28^{\circ} 49^{\prime} 22^{\prime \prime} \mathrm{S}, 49^{\circ} 14^{\prime} 43^{\prime \prime} \mathrm{W}$ & $2.8 \mathrm{~km}^{2}$ & $15 \mathrm{~h}$ & $\begin{array}{l}\text { Oct. } 2012 \text { to Jan. 2013, Aug. 2013, Oct. 2013, } \\
\text { Jan. 2016, Jan. } 2017\end{array}$ & ar, ex, la, ma \\
\hline 4. Foz do Rio Araranguá, Araranguá & $28^{\circ} 53^{\prime} 33^{\prime \prime} S, 49^{\circ} 18^{\prime} 30^{\prime \prime} \mathrm{W}$ & $3.2 \mathrm{~km}^{2}$ & $30 \mathrm{~h}$ & Feb. 2014 to Jan. 2015, Dec. 2015, & ar, be, ex, la, ma, ng \\
\hline 5. Praia dos Golfinhos, Balneário Arroio do Silva & $28^{\circ} 59^{\prime} 44^{\prime \prime} \mathrm{S}, 49^{\circ} 26^{\prime} 26^{\prime \prime} \mathrm{W}$ & $2.8 \mathrm{~km}^{2}$ & $20 \mathrm{~h}$ & Dec. 2013 & ar, cf, ex, ma, ng, om, pa \\
\hline 6. Lagoa do Caverá, Araranguá/Sombrio & $29^{\circ} 02^{\prime} 38^{\prime \prime S}, 49^{\circ} 33^{\prime} 34^{\prime \prime} \mathrm{W}$ & $3.0 \mathrm{~km}^{2}$ & $14 \mathrm{~h}$ & Dec. 2013, Mar. and Jul. 2016 & fo, la, ma, om, pa \\
\hline 7. Furnas - Lagoa do Sombrio, Sombrio & $29^{\circ} 07^{\prime} 39^{\prime \prime} \mathrm{S}, 49^{\circ} 39^{\prime} 42^{\prime \prime} \mathrm{W}$ & $1.2 \mathrm{~km}^{2}$ & $15 \mathrm{~h}$ & Mar., Apr. and Jun. 2015 & $\mathrm{cf}, \mathrm{fo}, \mathrm{la}, \mathrm{ma}, \mathrm{om}, \mathrm{pa}$, ua \\
\hline 8. Rio Novo - Lagoa do Sombrio, Balneário Gaivota & $29^{\circ} 09^{\prime} 56^{\prime \prime S}, 49^{\circ} 39^{\prime} 08^{\prime \prime} \mathrm{W}$ & $0.36 \mathrm{~km}^{2}$ & $44 \mathrm{~h}$ & Feb. to Apr. 2015, Jun. to Aug. 2015, Jul. 2016 & ar, cf, la, ma, ng, om, pa \\
\hline 9. Anita Garibaldi - Lagoa do Sombrio, Balneário Gaivota & $29^{\circ} 10^{\prime} 38^{\prime \prime S}, 49^{\circ} 39^{\prime} 56^{\prime \prime} \mathrm{W}$ & $0.28 \mathrm{~km}^{2}$ & $30 \mathrm{~h}$ & Sep. to Dec. 2014, Jul. 2015 & $\mathrm{ar}, \mathrm{cf}, \mathrm{fo}, \mathrm{la}, \mathrm{ma}, \mathrm{om}, \mathrm{pa}$ \\
\hline 10. Morro dos Macacos, Passo de Torres & $29^{\circ} 14^{\prime} 45^{\prime \prime S}, 49^{\circ} 43^{\prime} 39^{\prime \prime} \mathrm{W}$ & $1.9 \mathrm{~km}^{2}$ & $8 \mathrm{~h}$ & Mar. and Aug. 2015 & fo, la, ma, pa \\
\hline
\end{tabular}

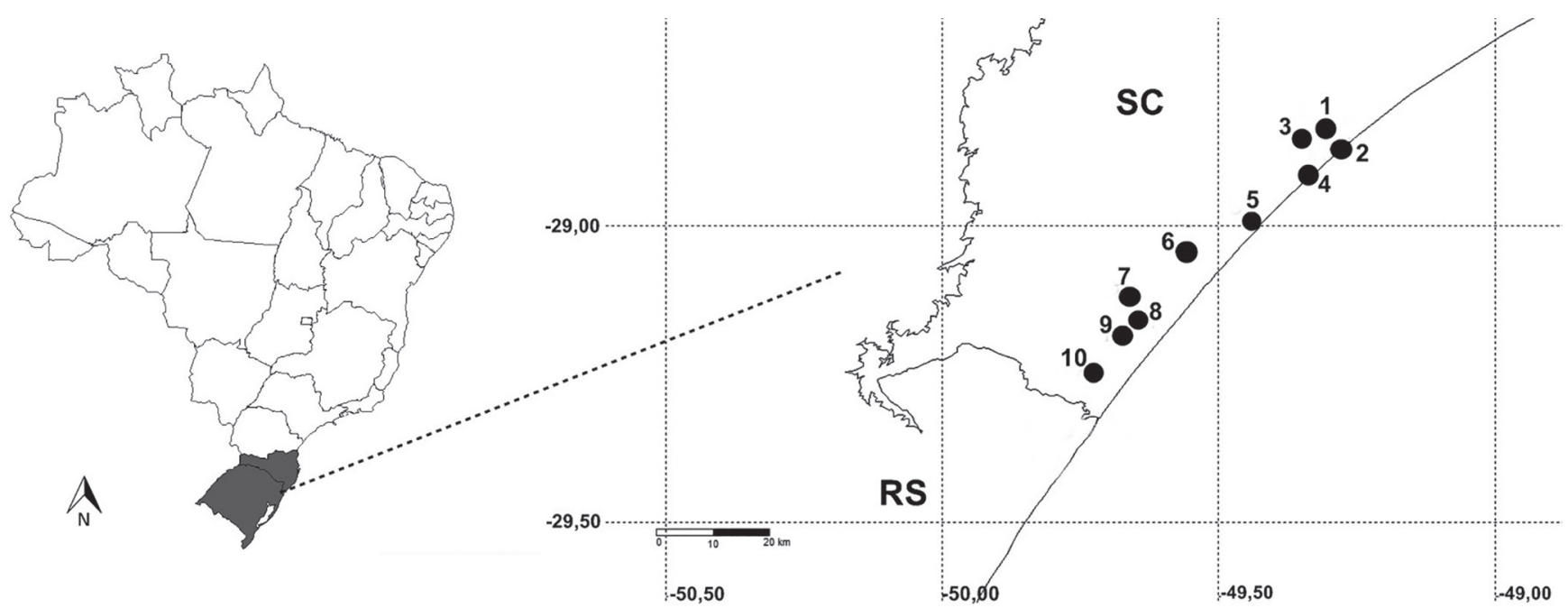

Figure 1. Localities surveyed in the southern coast of Santa Catarina state, Brazil. (1) Lagoa da Urussanga Velha; (2) Praia do Rincão; (3) Lagoa do Rincão/Jacaré; (4) Foz do Rio Araranguá; (5) Praia dos Golfinhos; (6) Lagoa do Caverá; (7) Furnas; (8) Rio Novo; (9) Anita Garibaldi; (10) Morro dos Macacos.

collecting the holotype of Sterna hirundinacea Lesson, 1831 in Laguna (Carlos \& Voisin, 2013) and recording an enigmatic and unidentified blue macaw in Imbituba (Straube, 2010). After Saint-Hilaire, some few naturalists visited the region to collect specimens which are roughly listed in classical catalogues (e.g., Hellmayr, 1936, Pinto, 1938). Later surveys in such area include broad-scale inventories with overall descriptions of the species and their distribution, which are to date the most complete studies on the avifauna for the southern coast of Santa Catarina (Rosário-Bege \& Marterer, 1991; Rosário, 1996). However, comprehensive studies are still missing for the last three decades, when the region experienced fast increasing habitat modification (Guadagnin \& Laidner, 1999). The few recent studies include bird inventories in beaches and islands in Laguna (Silva, 2003) and in the microbasin of the Ibiraquera lagoon (Piacentini \& Campbell-Thompson, 2006), and shorebirds censuses near the mouth of the Araranguá river (Branco et al., 2004). Additionally, several recent records of single or few species have been reported for the region (Azevedo
\& Ghizoni-Jr., 2005; Piacentini et al., 2006, 2009; Amorim \& Piacentini, 2006, 2007; Ghizoni-Jr. \& Azevedo, 2010; Willrich et al., 2015).

Here we carried out bird inventories in ten sites in the southern coast of Santa Catarina. We provide a qualitative checklist of birds, highlighting endemic and Nearctic, Austral and Neotropical migrants and provide data on habitats, abundances and sites of occurrence for threatened, novel and poorly known species for Santa Catarina. Based on our findings we discuss the importance of the region for bird conservation.

\section{MATERIALS AND METHODS}

\section{Study area}

The entire coastal region of southern Santa Catarina state extends from the municipalities of Garopaba to Passo de Torres, in the border with Rio Grande do Sul state (Guadagnin \& Laidner, 1999). In this study, we in- 
ventoried areas in the so called 'Complexo Lagunar de Sombrio' (sensu Burger, 1999; Guadagnin \& Laidner, 1999) which is a mosaic of wetlands ranging from 'Barra do Camacho' in the municipality of Jaguaruna $\left(28^{\circ} 36^{\prime} 55^{\prime \prime} \mathrm{S}, 48^{\circ} 51^{\prime} 30^{\prime \prime} \mathrm{W}\right)$ to Mampituba river in Passo de Torres (29 $19^{\prime} 29^{\prime \prime} \mathrm{S}, 49^{\circ} 42^{\prime} 46^{\prime \prime} \mathrm{W}$ ) (Fig. 1) (Table 1). In total, this region encompasses c. 25 lagoons, including Sombrio (50.6 km² of water surface), Caverá (3.50 km²), Jaguaruna $\left(3.25 \mathrm{~km}^{2}\right)$, Esteves $\left(2.90 \mathrm{~km}^{2}\right)$ and Urussanga Velha (2.35 km²) lagoons (Rosário-Bege \& Marterer, 1991).

The original landscape in this region was composed by mosaics of lowland forests, wetlands, restingas, grasslands, lagoons and oceanic sandy beaches (Teixeira et al.,
1986; Guadagnin \& Laidner, 1999) (Fig. 2). Lowland forests are 'dense ombrophilous forests', rich in epiphytes, lianas, ferns and with dominant emergent tree species like Ficus cestrifolia Schott, Syagrus romanzoffiana (Cham.) and Handroanthus umbellatus (Sond.) (Teixeira et al., 1986); marshes occur mainly along the lagoons and are composed by stands of emergent macrophytes as Scirpus giganteus Kunth, Schoenoplectus californicus (C.A. Mey.) Soják and Typha domingensis Pers. and floating macrophytes as Pistia stratiotes L. and Salvinia spp. (Teixeira et al., 1986); 'restingas' occur in sandy areas and hold mainly xeromorphic arbustive and arboreous plant species and the ground bromeliad Bromelia antiacan-
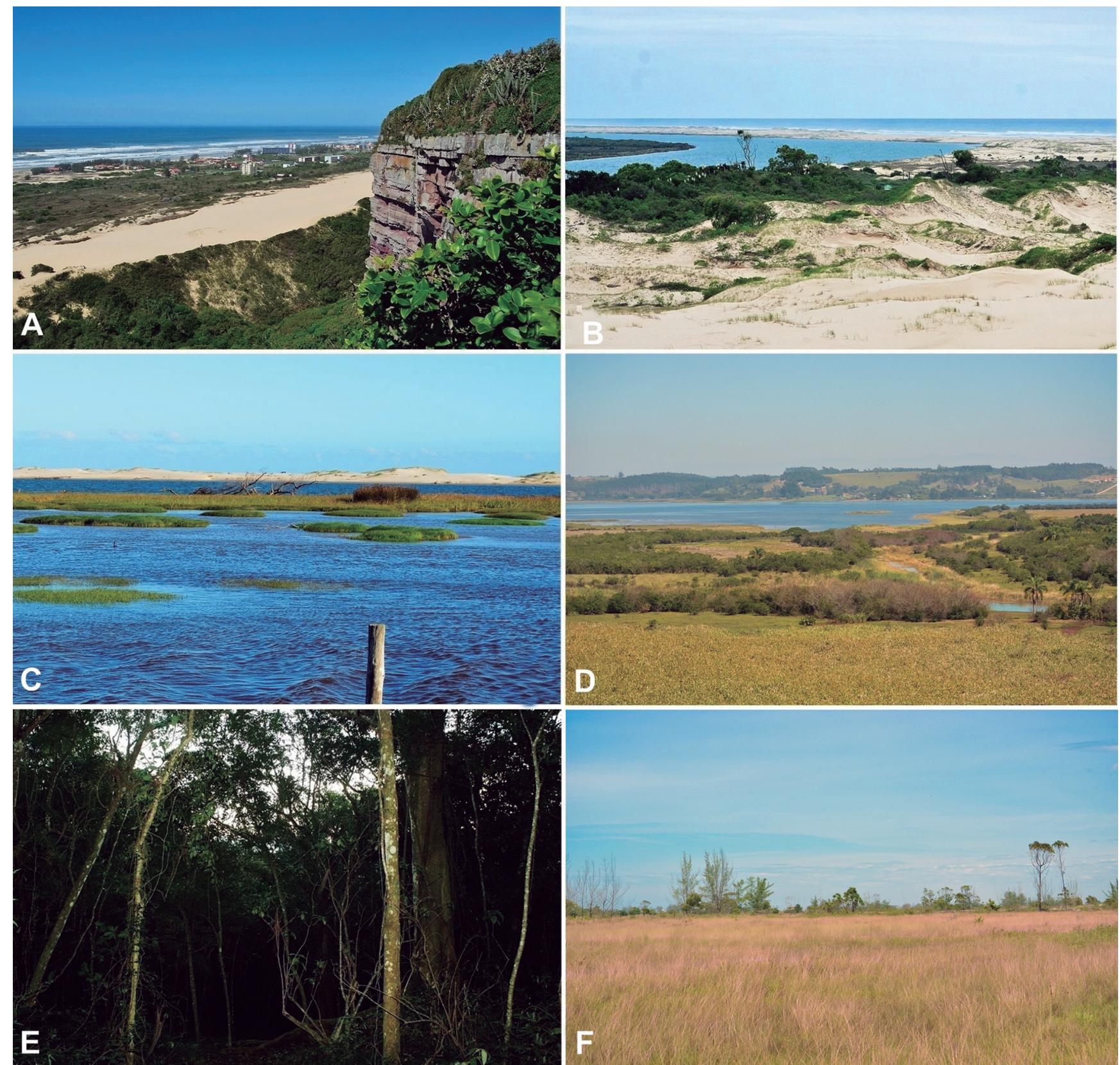

Figure 2. Habitats surveyed in the southern coast of Santa Catarina state, Brazil. (A) A general view of Morro dos Conventos beach, Araranguá; (B) Dunes and shrubby restingas near the mouth of Araranguá river, Araranguá; (C) Lagoon in the locality of llhas, near the mouth of Araranguá river, Araranguá; (D) A general view of the northeastern portion of Lagoa da Urussanga Velha, Balneário Rincão; (E) Understory of a lowland forest in Morro dos Macacos ecological park, Passo de Torres; (F) Wetlands of Typha domingensis in the border of Lagoa do Sombrio, Passo de Torres; (G) Mouth of Urussanga river, Balneário Rincão; (H) Wetlands with Scirpus giganteus in Lagoa do Rincão, Balneário Rincão. 
tha Bertol. (Teixeira et al., 1986; Falkenberg, 1999); natural grasslands are dominated by short to medium-sized Poaceae, Asteraceae and Cyperaceae and occur sparsely in sandy areas, specially amidst dunes (Klein et al., 2007; Menezes et al., 2015); lagoons overall have shallow waters with or without periodic seawater discharge and are surrounded by floating macrophytes, occurring as open marshes; oceanic sandy beaches have Pleistocenic and Holocenic origins, plant communities occur in frontal dunes and are composed essentially of Blutaparon portulacoides (A. St.-Hil.), Panicum racemosum (P. Beauv.) and Ipomoea pes-caprae (L.) R. Br and three rivers (Urussanga, Araranguá and Mampituba) flow though these areas to the ocean. Importantly, considerable portions of such natural habitats were replaced by cultivated fields (mainly rice and corn), planted pastures, urban areas and plantations of the exotic trees Eucalyptus spp. and Pinus spp. (Guadagnin \& Laidner, 1999). Climate is subtropical humid without dry seasons with annual average temperature of $19.8^{\circ} \mathrm{C}$, high relative humidity (85\%) and annual rainfall between 1.250 and $1.400 \mathrm{~mm}$ (Giannini et al., 2007).

\section{Data collection}

We sampled ten sites located from the mouth of Urussanga River to the mouth of Mampituba River (Fig. 1). Sampled areas varied from 0.28 to $4.75 \mathrm{~km}^{2}$ (summing up $21 \mathrm{~km}^{2}$ ) and up to $50 \mathrm{~m}$ above the sea level. Surveys were carried out between October 2012 and March 2018 and consisted of one-day samplings, lasting from 3 to $10 \mathrm{~h}$ per site, mainly from 07:00-12:00 h and 14:00-18:00 h. In total, each site was sampled between $8 \mathrm{~h}$ and $70 \mathrm{~h}$, summing up 274 hours of fieldwork. Coordinates, dates of field expeditions and habitats sampled in each site are described in Table 1. In each field expedition, all birds heard or seen with aid of binoculars in pre-existing trails and roads were identified. Playbacks of voice-recordings of species potentially occurring in the area were used, especially to search for cryptic species. Photographs and voice-recordings obtained during fieldwork were archived in the website WikiAves (www.wikiaves.com.br) and can be accessed online using voucher codes provided in Table 2. In order to provide a more complete inventory, species undetected during field work but with previous records for these sites in the literature (i.e., Rosário, 1996; Branco et al., 2004; Rupp et al., 2007) or WikiAves (searched up to July 2017) were included as Appendix. Data from Rosário (1996) was obtained in the website Aves de Santa Catarina (http://avesdesantacatarina.com. $\underline{\mathrm{br}}$. Also, some records available at WikiAves or made by other reseachers in the study areas were cited in the species account and/or in the discussion.

\section{Classification of endemic, migratory, threatened species and novel records}

Classification of endemic species for Atlantic Forest and Pampas follows Bencke et al. (2006). Migrant species followed the list of migratory birds of Brazil (Somenzari et al., 2018) and were classified in three categories as follows: "Nearctic migrants" breed in the Northern Hemisphere and move southward to spend the austral winter (Chesser, 1994); "Austral migrants" breed in austral regions of South America and move northward to spend the austral winter (Chesser, 1994) and; "Neotropical migrants" breed in our study area and move northward to spend the austral winter in tropical humid regions (Belton, 1994). We considered species of conservation concern those listed in at least one redlist in global (GL) (IUCN, 2015), national (BR) (MMA, 2014) and/or local (SC) levels (CONSEMA, 2011). For threatened species and distributional novelties, we provide details on number of individuals, habitats and dates of records. We considered distributional novelties in Santa Catarina as those species whose nearest record lies over $100 \mathrm{~km}$ from our study sites or species previously unknown or known from less than five localities in this state (i.e., Rosário-Bege \& Marterer, 1991; Rosário, 1996; Amorim \& Piacentini, 2006; Piacentini et al., 2006; Ghizoni-Jr. \& Azevedo, 2010). Both criteria follow Vizentin-Bugoni et al. (2015). Taxonomic nomenclature follows Piacentini et al. (2015). Specimens found dead were deposited at the ornithological collection of the Pontifícia Universidade Católica do Rio Grande do Sul.

\section{RESULTS}

Overall, we recorded 229 species encompassing 63 taxonomic families during field surveys (Table 2). Digital vouchers provide documentation of 198 species $(86 \%$ of the total). Four specimens found dead were deposited at the ornithological collection of the Pontifícia Universidade Católica do Rio Grande do Sul (MCP), namely Calonectris borealis (Cory, 1881) (entry number \#5081), Puffinus puffinus (Brünnich, 1764) (MCP-4627), Sterna cf. hirundinacea (entry number \#5089) - all three from Praia do Rincão - and Sturnella superciliaris (Bonaparte, 1850) (MCP-4621) from Lagoa do Caverá. In addition, 22 species were added to the list through literature search and WikiAves records (Appendix), summing 250 species to the study area.

\section{Endemic species}

Ten species recorded are endemic to the Atlantic Forest, namely Ortalis squamata (Lesson, 1829), Thalurania glaucopis (Gmelin, 1788), Picumnus temminckii Lafresnaye, 1845, Veniliornis spilogaster (Wagler, 1827), Myrmotherula unicolor (Ménétriès, 1835), Phacellodomus ferrugineigula (Pelzeln, 1858), Phylloscartes kronei Willis \& Oniki, 1992, Attila rufus (Vieillot, 1819), Tachyphonus coronatus (Vieillot, 1822) and Habia rubica (Vieillot, 1817). The only species endemic to Pampas was Limnornis curvirostris Gould, 1839. 
Table 2. Bird species recorded at ten sites in the southern coast of Santa Catarina state, Brazil. Details on localities of record, habitat, status of conservation, endemism, migration and voucher are provided. " + " indicates species new to the southern coast of Santa Catarina in relation to the two previous broad-scale inventories (Rosário-Bege \& Marterer, 1991; Rosário, 1996). Localities: $1=$ Lagoa da Urussanga Velha, $2=$ Praia do Rincão, $3=$ Lagoa do Rincão/Jacaré, $4=$ Foz do Rio Araranguá, $5=$ Praia dos Golfinhos, $6=$ Lagoa do Caverá, $7=$ Furnas, $8=$ Rio Novo, $9=$ Anita Garibaldi, $10=$ Morro dos Macacos. Habitat: be $=$ beach, $\mathrm{cf}=$ cultivated field, ex = plantation of exotic trees - eucalyptus or pinus, $\mathrm{fl}=$ fly over, $\mathrm{fo}_{0}=$ lowland forest, la $=$ coastal lagoon, ma $=$ marsh with stands of emergent macrophytes, $\mathrm{ng}=$ natural grassland, $\mathrm{om}=$ open marsh, $\mathrm{pa}=$ planted pasture, $\mathrm{sr}=$ shrubby restinga, $\mathrm{ua}=$ urban area. Status: Atl = species endemic to the Atlantic Forest, Pam = species endemic to the Pampas, $\mathrm{Cr}=$ critically endangered, $\mathrm{En}=$ endangered, $\mathrm{Vu}=$ vulnerable, $\mathrm{Nt}=$ near threatened, $\mathrm{GL}=$ global level, $\mathrm{BR}=$ national level, $\mathrm{SC}=$ state level, $\mathrm{Nm}=$ Nearctic migrant, $\mathrm{Am}=$ Austral migrant, $\mathrm{Tm}=$ Neotropical migrant. Photo/Voice-recording: catalogue numbers of our digital vouchers available at the website WikiAves.

\begin{tabular}{|c|c|c|c|c|}
\hline Taxon & Localities & Habitat & Status & Photo/Voice-recording \\
\hline \multicolumn{5}{|l|}{ Tinamidae Gray, 1840} \\
\hline Crypturellus obsoletus (Temminck, 1815) + & 6 & fo & & WA2045021 \\
\hline Nothura maculosa (Temminck, 1815) & $1,2,4,5,6,7,8,9,10$ & $\mathrm{cf}, \mathrm{ng}, \mathrm{pa}$ & & WA916393 \\
\hline \multicolumn{5}{|l|}{ Anhimidae Stejneger, 1885} \\
\hline Chauna torquata (0ken, 1816) & $5,6,7,8,9,10$ & om & & WA1178200, WA1495224, WA1744286, WA1749013 \\
\hline \multicolumn{5}{|l|}{ Anatidae Leach, 1820} \\
\hline Dendrocygna viduata (Linnaeus, 1766) & $1,2,3,4,5,6,7,8,9,10$ & la, om & & WA1586936, WA1804435 \\
\hline Amazonetta brasiliensis (Gmelin, 1789) & $1,2,3,4,5,6,7,8,9,10$ & la, om & & WA1739496, WA1808835, WA1809421 \\
\hline Anas georgica Gmelin, 1789 & 2,4 & $\mathrm{la}, \mathrm{om}$ & & WA1620614, WA1805133 \\
\hline Anas versicolor Vieillot, 1816 & $4,7,8$ & $\mathrm{la}, 0 \mathrm{~m}$ & & WA1082946, WA1739498 \\
\hline \multicolumn{5}{|l|}{ Podicipedidae Bonaparte, 1831} \\
\hline Rollandia rolland (Quoy \& Gaimard, 1824) & 1,3 & la & & WA1215451 \\
\hline Podilymbus podiceps (Linnaeus, 1758) + & $1,3,10$ & la & & WA1082949, WA1809423 \\
\hline Podicephorus major (Boddaert, 1783) & 2,4 & la & & WA1461291 \\
\hline \multicolumn{5}{|l|}{ Spheniscidae Bonaparte, 1831} \\
\hline Spheniscus magellanicus (Forster, 1781) & 2 & be & $\mathrm{Am}, \mathrm{Nt}-\mathrm{GL}$ & WA988659 \\
\hline \multicolumn{5}{|l|}{ Diomedeidae Gray, 1840} \\
\hline Thalassarche chlororhynchos (Gmelin, 1789) + & 2 & be & Am, En-GL, BR, SC & \\
\hline Thalassarche melanophris (Temminck, 1828) & 2 & be & Am, Nt-GL, En-SC & \\
\hline \multicolumn{5}{|l|}{ Procellaridae Leach, 1820} \\
\hline Procellaria aequinoctialis Linnaeus, 1758 & 2 & be & $\mathrm{Am}, \mathrm{Vu}-\mathrm{GL}, \mathrm{BR}, \mathrm{SC}$ & \\
\hline Calonectris borealis (Cory, 1881) & 2 & be & $\mathrm{Nm}$ & \\
\hline Puffinus puffinus (Brünnich, 1764) & 2 & be & $\mathrm{Nm}$ & \\
\hline \multicolumn{5}{|l|}{ Cracidae Rafinesque, 1815} \\
\hline Ortalis squamata (Lesson, 1829) & $1,4,5,8,9$ & $f_{0}$ & Atl & WA1740948, WA2354730 \\
\hline \multicolumn{5}{|l|}{ Ciconiidae Sundevall, 1836} \\
\hline Ciconia maguari (Gmelin, 1789) & $4,5,6,7,8,9,10$ & om & & WA1631472 \\
\hline Mycteria americana Linnaeus, 1758 + & 7 & om & & \\
\hline \multicolumn{5}{|l|}{ Fregatidae Degland \& Gerbe, 1867} \\
\hline Fregata magnificens Mathews, 1914 & $1,2,3,4,6,9$ & $\mathrm{fl}$ & & WA1987245 \\
\hline \multicolumn{5}{|l|}{ Phalacrocoracidae Reichenbach, 1849} \\
\hline Nannopterum brasilianus (Gmelin, 1789) & $1,2,3,4,5,6,7,8,9,10$ & be, la & & WA1172630, WA1216379 \\
\hline \multicolumn{5}{|l|}{ Ardeidae Leach, 1820} \\
\hline Tigrisoma lineatum (Boddaert, 1783) + & 9 & om & & WA1587910 \\
\hline Botaurus pinnatus (Wagler, 1829) + & $3,7,8,9$ & ma, om & & WA1667250 \\
\hline Ixobrychus involucris (Vieillot, 1823) & $6,8,9$ & ma & & WA1256664 \\
\hline Nycticorax nycticorax (Linnaeus, 1758) & $1,2,4,5,6,7,8$ & be, om & & WA1586440 \\
\hline Butorides striata (Linnaeus, 1758) & $1,2,3,4,5,6,7,9$ & la, om & & WA1496237, WA2047195 \\
\hline Bubulcus ibis (Linnaeus, 1758) & $1,2,3,4,5,6,7,8,9,10$ & $\mathrm{cf}, \mathrm{ng}, \mathrm{pa}$ & & WA1740945, WA1805136 \\
\hline Ardea cocoi Linnaeus, 1766 & $1,2,4,6,7,8,9$ & be, om & & WA1026574 \\
\hline Ardea alba Linnaeus, 1758 & $1,2,3,4,5,6,7,8,9,10$ & be, om & & WA1987261 \\
\hline Syrigma sibilatrix (Temminck, 1824) & $1,2,3,4,5,6,7,8,9,10$ & $\mathrm{cf}, \mathrm{ng}, \mathrm{om}, \mathrm{pa}$ & & WA1497322 \\
\hline Egretta thula (Molina, 1782) & $1,2,4,5,7,10$ & be, om & & WA1026576, WA1740927 \\
\hline \multicolumn{5}{|l|}{ Threskiornithidae Poche, 1904} \\
\hline Plegadis chihi (Vieillot, 1817) & $1,2,3,4,5,6,7,8,9,10$ & om & & \\
\hline Phimosus infuscatus (Lichtenstein, 1823) + & $1,2,3,4,5,6,7,8,9,10$ & be, ng, om, pa, ua & & WA1620617, WA1631382 \\
\hline Theristicus caudatus (Boddaert, 1783) & $1,2,3,4,5,6,7,8,9,10$ & $\mathrm{cf}, \mathrm{ng}, \mathrm{pa}, \mathrm{ng}$ & & WA1215452 \\
\hline Platalea ajaja Linnaeus, 1758 & 4,9 & om & & WA1444654, WA1805134 \\
\hline \multicolumn{5}{|l|}{ Cathartidae Lafresnaye, 1839} \\
\hline Cathartes aura (Linnaeus, 1758) & $1,2,3,4,5,6,7,8,9,10$ & be, fl & & WA1026575 \\
\hline
\end{tabular}


Taxon
Cathartes burrovianus Cassin, 1845

Coragyps atratus (Bechstein, 1793)

Accipitridae Vigors, 1824

Elanus leucurus (Vieillot, 1818) +

Circus buffoni (Gmelin, 1788)

Accipiter striatus Vieillot, 1808

Rostrhamus sociabilis (Vieillot, 1817)

Heterospizias meridionalis (Latham, 1790)

Rupornis magnirostris (Gmelin, 1788)

Aramidae Bonaparte, 1852

Aramus guarauna (Linnaeus, 1766)

Rallidae Rafinesque, 1815

Aramides ypecaha (Vieillot, 1819) +

Aramides saracura (Spix, 1825)

Laterallus melanophaius (Vieillot, 1819)

Laterallus leucopyrrhus (Vieillot, 1819)

Mustelirallus albicollis (Vieillot, 1819)

Pardirallus nigricans (Vieillot, 1819)

Pardirallus sanguinolentus (Swainson, 1838)

Gallinula galeata (Lichtenstein, 1818)

Fulica armillata Vieillot, 1817

Charadriidae Leach, 1820

Vanellus chilensis (Molina, 1782)

Pluvialis dominica (Statius Muller, 1776)

Pluvialis squatarola (Linnaeus, 1758)

Charadrius semipalmatus Bonaparte, 1825

Charadrius collaris Vieillot, 1818

Charadrius falklandicus Latham, $1790+$

Charadrius modestus Lichtenstein, 1823

Haematopodidae Bonaparte, 1838

Haematopus palliatus Temminck, 1820

Recurvirostridae Bonaparte, 1831

Himantopus melanurus Vieillot, 1817

Scolopacidae Rafinesque, 1815

Gallinago paraguaiae (Vieillot, 1816)

Gallinago undulata (Boddaert, 1783)

Tringa melanoleuca (Gmelin, 1789)

Tringa semipalmata (Gmelin, 1789) +

Tringa flavipes (Gmelin, 1789)

Calidris canutus (Linnaeus, 1758)

Calidris alba (Pallas, 1764)

Calidris pusilla (Linnaeus, 1766)

Calidris fuscicollis (Vieillot, 1819)

Calidris melanotos (Vieillot, 1819) +

Jacanidae Chenu \& Des Murs, 1854

Jacana jacana (Linnaeus, 1766)

Laridae Rafinesque, 1815

Chroicocephalus maculipennis (Lichtenstein, 1823)

Larus atlanticus Olrog, $1958+$

Larus dominicanus Lichtenstein, 1823

Sternidae Vigors, 1825

Sternula superciliaris (Vieillot, 1819)

Sterna hirundo Linnaeus, $1758+$

Sterna hirundinacea Lesson, 1831

Sterna trudeaui Audubon, 1838

Thalasseus acuflavidus (Cabot, 1847)

Thalasseus maximus (Boddaert, 1783)

Rynchopidae Bonaparte, 1838

Rynchops niger Linnaeus, 1758

Stercorariidae Gray 1870

$1,3,6,8$

$1,2,3,4,5,6,7,8,9,10$

Habitat

pa

be, fl, ua

9

$1,2,3,4,5,6,7,8,9$

6

$2,3,6,7,8$

$1,2,5,8,10$

$1,2,3,4,5,6,7,8,9,10$

$2,3,4,5,6,7,8,9,10$

$7,8,9,10$

$2,6,8,9,10$

$1,3,5,6,7,8,9,10$$$
1
$$

$1,2,4,5,9$

$2,5,6,7,8,9,10$

$1,2,3,4,6,7,8,9$

$2,3,5,6,7,8,9$

2

$1,2,3,4,5,6,7,8,9,10$

2,4

2,4

2,4

2,4

2

2

$1,2,4$

$1,2,4,6,7,10$

$1,2,3,4,5,6,7,8,9$

2

2,4

4

$1,2,4$

2,4

2,4

2

2,4

2

$1,2,3,4,5,6,7,8,9,10$

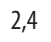

2

2,4

2,4

2,4

2,4

2,4

2,4

2,4

2,4 pa

ma, om

fo

ma, om

$\mathrm{ng}, \mathrm{pa}$

ex, fo, ua

ma, om

ma, om

ma

ma

ma, om

ma, om

ma, om

la, om

be, la

cf, ng, om, pa, ua

be $\quad \mathrm{Nm}$

be $\quad \mathrm{Nm}$

be $\quad \mathrm{Nm}$

be

be

be

be, la

be, la, om

om

ng

be, la

be

be, la

be

be

be

be

be

la, om

be

be

be

be

be

be

$\mathrm{Nm}$

Vu-BR

be

be

be

En-BR, Vu-SC
Photo/Voice-recording

WA1172631, WA1603881, WA1976484,

WA1027470, WA1739507

WA1496238, WA1621757, WA1631384 WA2209115, WA2229797

WA1803625

WA1215399, WA1976489

WA1497320

WA1178201, WA1805142

WA1495195, WA1496255, WA1634969, WA1739497 WA843617

WA1016183, WA1749121

WA2354729

WA1068010, WA1082947

WA1027626, WA1667279

WA1740947, WA1805144

WA1214653

WA1576083, WA1744217

WA1805145

WA1331389

WA1024468

WA1331385

WA965980, WA1372703

WA1576074

WA1621759

WA1214763, WA1744231

WA1744210

WA940366

WA1331388

WA874975

WA1576078

WA1576082

WA2927732

WA1215450

WA1675808

WA1603878

WA965981

WA1372730, WA1371318

WA804253

WA1805141, WA988658

WA1803622

WA807753, WA2079160

WA1207781, WA1216378

WA808747, WA911660, WA999158

WA988660, WA1622675

WA1572905, WA808745, WA911670, WA2079174 


\begin{tabular}{|c|c|c|c|c|}
\hline Taxon & Localities & Habitat & Status & Photo/Voice-recording \\
\hline \multicolumn{5}{|l|}{ Columbidae Leach, 1820} \\
\hline Columba livia Gmelin, 1789 & $2,3,7,8,10$ & be, ua & & WA2006330 \\
\hline Columbina talpacoti (Temminck, 1810) & $1,2,3,4,5,6,7,8,9,10$ & $\mathrm{cf}, \mathrm{sr}$, ua & & WA1983693 \\
\hline Columbina picui (Temminck, 1813) & $1,2,3,4,5,6,7,8,9,10$ & $\mathrm{ng}, \mathrm{sr}$, ua & & WA886852, WA1497319 \\
\hline Patagioenas picazuro (Temminck, 1813) & $1,2,3,4,5,6,8,9$ & $c f, e x, f o, p a$, & & WA1745049 \\
\hline Zenaida auriculata (Des Murs, 1847) + & $1,2,3,4,5,6,7,8,9,10$ & cf, sr, ua & & WA1983694 \\
\hline Leptotila verreauxi Bonaparte, 1855 & $1,2,3,4,5,6,7,8,9,10$ & fo, sr & & WA1495244, WA1739487 \\
\hline \multicolumn{5}{|l|}{ Cuculidae Leach, 1820} \\
\hline Piaya cayana (Linnaeus, 1766) & $1,4,6,7,8,9,10$ & fo, sr & & WA1983701, WA1496257 \\
\hline Coccyzus melacoryphus Vieillot, $1817+$ & $1,2,8$ & $\mathrm{sr}$ & & WA1976486 \\
\hline Coccyzus americanus (Linnaeus, 1758) + & 6 & $\mathrm{sr}$ & $\mathrm{Nm}$ & WA2018654 \\
\hline Crotophaga ani Linnaeus, 1758 & $1,2,3,4,5,6,8,9,10$ & $\mathrm{pa}, \mathrm{sr}$, ua & & WA1983695 \\
\hline Guira guira (Gmelin, 1788) & $1,2,3,4,5,6,7,8,9,10$ & cf, ex, pa, sr, ua & & WA1591752, WA1804429 \\
\hline Tapera naevia (Linnaeus, 1766) & $1,2,3,4,5,6,8,9$ & sr & & WA1079735 \\
\hline \multicolumn{5}{|l|}{ Strigidae Leach, 1820} \\
\hline Bubo virginianus (Gmelin, 1788) + & 7,10 & fo & & WA1631386 \\
\hline Athene cunicularia (Molina, 1782) & $1,2,4,5,6,8$ & be, ng, pa & & WA1586933, WA891425, WA1172628, WA1804426 \\
\hline \multicolumn{5}{|l|}{ Caprimulgidae Vigors, 1825} \\
\hline Hydropsalis torquata (Gmelin, 1789) & 3 & $\mathrm{sr}$ & & \\
\hline Podager nacunda (Vieillot, 1817) + & 2,8 & pa & & WA1667313 \\
\hline \multicolumn{5}{|l|}{ Apodidae OIphe-Galliard, 1887} \\
\hline Cypseloides fumigatus (Streubel, 1848) + & $2,8,9$ & $\mathrm{fl}$ & & WA1452837, WA1216380 \\
\hline Streptoprocne zonaris (Shaw, 1796) & $1,2,5,7$ & $\mathrm{fl}$ & & WA1740949 \\
\hline Streptoprocne biscutata (Sclater, 1866) + & 2 & $\mathrm{fl}$ & & WA1983854 \\
\hline Chaetura meridionalis Hellmayr, 1907 & 2,9 & $\mathrm{fl}$ & & \\
\hline \multicolumn{5}{|l|}{ Trochilidae Vigors, 1825} \\
\hline Eupetomena macroura (Gmelin, 1788) + & 2,4 & ua & & WA1247525 \\
\hline Anthracothorax nigricollis (Vieillot, 1817) + & 2,4 & ua & & WA844048, WA843599 \\
\hline Chlorostilbon lucidus (Shaw, 1812) + & $1,2,3,4,5,6,7,9$ & fo, sr, ua & & WA843602, WA1634978 \\
\hline Thalurania glaucopis (Gmelin, 1788) + & 10 & fo & Atl & \\
\hline Leucochloris albicollis (Vieillot, 1818) & 2,5 & ua & & WA1239257 \\
\hline Amazilia fimbriata (Gmelin, 1788) + & $1,2,3,4,5,6$ & fo, sr, ua & & WA1586445, WA843603 \\
\hline \multicolumn{5}{|l|}{ Alcedinidae Rafinesque, 1815} \\
\hline Megaceryle torquata (Linnaeus, 1766) & $1,2,3,8,9$ & la & & WA1622681, WA2228829 \\
\hline Chloroceryle amazona (Latham, 1790) & 2,4 & la & & WA1496286 \\
\hline \multicolumn{5}{|l|}{ Picidae Leach, 1820} \\
\hline Picumnus temminckii Lafresnaye, 1845 & $1,2,4,6,8,10$ & fo, sr & Atl & WA1983699 \\
\hline Melanerpes candidus $(0 \mathrm{tto}, 1796)+$ & $1,2,6,7$ & $\mathrm{cf}, \mathrm{pa}$ & & WA1826616, WA1804271 \\
\hline Veniliornis spilogaster (Wagler, 1827) + & 1,9 & fo, sr & & WA1215453, WA1590761 \\
\hline Colaptes melanochloros (Gmelin, 1788) & 1,9 & sr & & \\
\hline Colaptes campestris (Vieillot, 1818) & $1,2,3,4,5,6,7,8,9,10$ & $\mathrm{cf}, \mathrm{ng}, \mathrm{pa}$ & & WA1804439 \\
\hline Celeus flavescens (Gmelin, 1788) & $1,4,5,6,8$ & fo, sr & & WA1215398, WA2044988 \\
\hline \multicolumn{5}{|l|}{ Falconidae Leach, 1820} \\
\hline Caracara plancus (Miller, 1777) & $1,2,3,4,5,6,7,8,9,10$ & be, ex, ng, pa, ex & & WA1572864, WA1622683 \\
\hline Milvago chimachima (Vieillot, 1816) & $1,2,3,4,5,6,7,8,9,10$ & be, ex, fo, sr & & WA1804428, WA1739510 \\
\hline Milvago chimango (Vieillot, 1816) & $1,2,3,4,5,6,7,8,9,10$ & be, ng, pa & & WA1557885, WA1603889, WA1809418 \\
\hline Herpetotheres cachinnans (Linnaeus, 1758) + & 4,5 & ex, sr & & WA1178257 \\
\hline Falco sparverius Linnaeus, 1758 & $2,4,5,9,10$ & $c f, n g, p a$, ua & & WA1027471, WA1215400 \\
\hline Falco femoralis Temminck, $1822+$ & $1,2,4,7,8$ & ng, pa & & WA1013623, WA1667276, WA1634980, WA1025231 \\
\hline \multicolumn{5}{|l|}{ Psittacidae Rafinesque, 1815} \\
\hline Myiopsitta monachus (Boddaert, 1783) + & $1,2,3,4,5,6,7,8,9,10$ & cf, ex, pa, ua & & WA1804427, WA1739506, WA901069 \\
\hline \multicolumn{5}{|l|}{ Thamnophilidae Swainson, 1824} \\
\hline Myrmotherula unicolor (Ménétriès, 1835) + & 6 & fo & Atl, Nt-GL & WA2045047 \\
\hline Thamnophilus ruficapillus Vieillot, 1816 & $1,2,3,4,8$ & $\mathrm{sr}$ & & WA1804273, WA988355 \\
\hline Thamnophilus caerulescens Vieillot, 1816 & $1,4,6,7$ & fo, sr & & WA1675785, WA1739493 \\
\hline \multicolumn{5}{|l|}{ Conopophagidae Sclater \& Salvin, 1873} \\
\hline Conopophaga lineata (Wied, 1831) + & $1,6,10$ & fo & & WA2045040 \\
\hline \multicolumn{5}{|l|}{ Scleruridae Swainson, 1827} \\
\hline Geositta cunicularia (Vieillot, 1816) & 2,4 & be, ng & Vu-SC & WA1014901, WA942792, WA1583389 \\
\hline \multicolumn{5}{|l|}{ Furnariidae Gray, 1840} \\
\hline Furnarius rufus (Gmelin, 1788) & $1,2,3,4,5,6,7,8,9,10$ & $c f, n g, p a$, ua & & WA2006331 \\
\hline
\end{tabular}




\begin{tabular}{|c|c|c|c|c|}
\hline Taxon & Localities & Habitat & Status & Photo/Voice-recording \\
\hline Limnornis curvirostris Gould, $1839+$ & 8,9 & ma & Pam & WA1668353, WA1296192, WA1452904 \\
\hline Phleocryptes melanops (Vieillot, 1817) & $2,4,6,7,8,9$ & ma & & WA1667249, WA1809443 \\
\hline Anumbius annumbi (Vieillot, 1817) & $1,2,4,5,6,7,8,9$ & $\mathrm{ng}, \mathrm{pa}$ & & WA1025232, WA1603879, WA891240, WA1588847 \\
\hline Phacellodomus ferrugineigula (Pelzeln, 1858) + & 1,8 & ma & Atl & WA1739509, WA1739483, WA2354732, WA2354763 \\
\hline Schoeniophylax phryganophilus (Vieillot, 1817) + & 6,9 & pa & & WA2045008 \\
\hline Certhiaxis cinnamomeus (Gmelin, 1788) & $1,2,3,4,5,6,7,8,9,10$ & ma & & WA1588848, WA1452835 \\
\hline Synallaxis spixi Sclater, 1856 & $1,3,4,5,7,8,9,10$ & $\mathrm{sr}$ & & \\
\hline \multicolumn{5}{|l|}{ Pipridae Rafinesque, 1815} \\
\hline Manacus manacus (Linnaeus, 1766) & 1,6 & fo & & \\
\hline Chiroxiphia caudata (Shaw \& Nodder, 1793) & 6 & fo & Atl & WA2228939 \\
\hline \multicolumn{5}{|l|}{ Platyrinchidae Bonaparte, 1854} \\
\hline Platyrinchus mystaceus Vieillot, $1818+$ & 6 & fo & & \\
\hline \multicolumn{5}{|l|}{ Tachurisidae Ohlson et al. 2013} \\
\hline Tachuris rubrigastra (Vieillot, 1817) & 8 & ma & Vu-SC & WA1749009, WA1739508, WA2228844 \\
\hline \multicolumn{5}{|l|}{ Rhynchocyclidae Berlepsch, 1907} \\
\hline Leptopogon amaurocephalus Tschudi, $1846+$ & 10 & fo & & \\
\hline Tolmomyias sulphurescens (Spix, 1825) + & 1 & fo & & \\
\hline Poecilotriccus plumbeiceps (Lafresnaye, 1846) & 1,6 & sr & & WA2045035 \\
\hline Phylloscartes kronei Willis \& Oniki, $1992+$ & $1,6,10$ & $\mathrm{sr}, \mathrm{fo}_{0}$ & Atl, Nt-GL & WA2045020, WA2231007 \\
\hline \multicolumn{5}{|l|}{ Tyrannidae Vigors, 1825} \\
\hline Camptostoma obsoletum (Temminck, 1824) & $1,2,3,4,5,6,7,8,9,10$ & $\mathrm{sr}, \mathrm{fo}$ & & \\
\hline Elaenia flavogaster (Thunberg, 1822) & $1,2,3,4,6,8,9,10$ & $\mathrm{sr}$ & & \\
\hline Elaenia parvirostris Pelzeln, 1868 & $1,3,4,9$ & $\mathrm{sr}, \mathrm{fo}$ & $\mathrm{Tm}$ & WA1495307, WA2354726 \\
\hline Elaenia obscura (d'Orbigny \& Lafresnaye, 1837) & $1,2,3,4,5,6,9$ & $\mathrm{Sr}$ & & WA2045031 \\
\hline Pseudocolopteryx sclateri (Oustalet, 1892) & $3,6,7,8,9$ & ma & & WA1256663, WA1631412, WA1739501, WA1587907 \\
\hline Pseudocolopteryx flaviventris (d'Orbigny \& Lafresnaye, 1837) + & 8,9 & ma & & WA1667277, WA1452903, WA1557931 \\
\hline Serpophaga nigricans (Vieillot, 1817) & $5,7,8,9$ & ma & & WA1497321, WA1667253, WA1587908, WA1593543 \\
\hline Serpophaga subcristata (Vieillot, 1817) + & $1,2,3,4,8,9$ & sr & & WA1495271, WA1027469 \\
\hline Attila rufus (Vieillot, 1819) + & 6,10 & fo & Atl & WA2219896, WA2229809 \\
\hline Myiarchus swainsoni Cabanis \& Heine, 1859 & 1 & fo & $\mathrm{Tm}$ & WA2354836 \\
\hline Pitangus sulphuratus (Linnaeus, 1766) & $1,2,3,4,5,6,7,8,9,10$ & cf, ex, fo, sr, ua & & WA1744717, WA1745018 \\
\hline Machetornis rixosa (Vieillot, 1819) & $1,2,3,4,5,6,7,8,9,10$ & ng, pa, ua & & WA1667382, WA1452838, WA1631381 \\
\hline Myiodynastes maculatus (Statius Muller, 1776) + & 8 & fo & $\mathrm{Tm}$ & \\
\hline Tyrannus melancholicus Vieillot, 1819 & $1,2,3,4,5,6,7,8,9,10$ & $f_{0}, s r$ & $\mathrm{Tm}$ & WA1983709, WA1594760 \\
\hline Tyrannus savana Daudin, 1802 & $1,2,3,4,6,7$ & $\mathrm{cf}, \mathrm{ng}, \mathrm{pa}, \mathrm{sr}$ & $\mathrm{Tm}$ & WA1803623 \\
\hline Empidonomus varius (Vieillot, 1818) + & $1,2,9$ & $\mathrm{fo}_{\mathrm{s}} \mathrm{sr}$ & Tm & WA1496256, WA1591751 \\
\hline Myiophobus fasciatus (Statius Muller, 1776) & $1,4,5,6,8$ & $\mathrm{sr}$ & & WA1215397, WA1496241, WA1983698, WA1591754 \\
\hline Arundinicola leucocephala (Linnaeus, 1764) & $6,7,8,9$ & ma & & WA1809440, WA1667381, WA1603880, WA1590782 \\
\hline Cnemotriccus fuscatus (Wied, 1831) + & 10 & fo & & \\
\hline Lathrotriccus euleri (Cabanis, 1868) + & 6 & fo & $\mathrm{Tm}$ & \\
\hline Hymenops perspicillatus (Gmelin, 1789) & $2,8,9$ & ma, ng, om & & WA1013625, WA1024351 \\
\hline Satrapa icterophrys (Vieillot, 1818) & $1,2,3,4,7,8,9$ & sr & & WA1634834, WA1983710, WA1593541 \\
\hline Xolmis irupero (Vieillot, 1823) & $1,2,3,4,5,6,7,8,9,10$ & $\mathrm{ng}, \mathrm{pa}$ & & WA1622676, WA1631471, WA1603887 \\
\hline Xolmis dominicanus (Vieillot, 1823) & 6 & pa & Vu-GL-BR, En-SC & WA2208102, WA2209035, WA2209036 \\
\hline \multicolumn{5}{|l|}{ Vireonidae Swainson, 1837} \\
\hline Cyclarhis gujanensis (Gmelin, 1789) & $1,5,6,7,8,9,10$ & $f_{0}, \mathrm{sr}$ & & WA2354727 \\
\hline Vireo chivi (Vieillot, 1817) + & $1,2,5,9$ & fo & $\mathrm{Tm}$ & WA2354734 \\
\hline \multicolumn{5}{|l|}{ Hirundinidae Rafinesque, 1815} \\
\hline Pygochelidon cyanoleuca (Vieillot, 1817) & $1,2,3,4,5,6,7,8,9,10$ & be, ng, pa, ua & & \\
\hline Alopochelidon fucata (Temminck, 1822) & $2,3,4,8,10$ & be, ng, pa & & WA1620613 \\
\hline Stelgidopteryx ruficollis (Vieillot, 1817) & 1 & pa & $\mathrm{Tm}$ & WA1976493 \\
\hline Progne tapera (Vieillot, 1817) & $1,2,3,4,5,6,7,8,9,10$ & $\mathrm{cf}, \mathrm{ng}, \mathrm{pa}$ & $\mathrm{Tm}$ & WA1583590 \\
\hline Progne chalybea (Gmelin, 1789) & $1,2,3,4,7$ & cf, ng, ua & $\mathrm{Tm}$ & WA807751 \\
\hline Tachycineta leucorrhoa (Vieillot, 1817) & $1,2,3,4,5,6,7,8,9,10$ & be, ng, pa & & WA844003 \\
\hline Hirundo rustica Linnaeus, 1758 & $1,2,4,8,9,10$ & $\mathrm{ng}, \mathrm{pa}$ & $\mathrm{Nm}$ & WA1803624 \\
\hline \multicolumn{5}{|l|}{ Troglodytidae Swainson, 1831} \\
\hline Troglodytes musculus Naumann, 1823 & $1,2,3,4,5,6,7,8,9,10$ & $\mathrm{Sr}, \mathrm{ua}$ & & WA2219900 \\
\hline \multicolumn{5}{|l|}{ Polioptilidae Baird, 1858} \\
\hline Polioptila dumicola (Vieillot, 1817) + & $2,7,8,9$ & $\mathrm{sr}$ & & WA843574, WA1495243, WA1593539, WA2228871 \\
\hline \multicolumn{5}{|l|}{ Turdidae Rafinesque, 1815} \\
\hline Turdus flavipes Vieillot, $1818+$ & 4,6 & $f_{0}$ & & \\
\hline
\end{tabular}




\begin{tabular}{|c|c|c|c|c|}
\hline Taxon & Localities & Habitat & Status & Photo/Voice-recording \\
\hline Turdus leucomelas Vieillot, $1818+$ & $1,4,6$ & fo & & WA2229159, WA2354733 \\
\hline Turdus rufiventris Vieillot, 1818 & $1,2,3,4,5,6,7,8,9,10$ & fo, sr, ua & & WA2229124 \\
\hline Turdus amaurochalinus Cabanis, 1850 & $1,2,3,4,5,6,7,8,9,10$ & $\mathrm{fo}_{\mathrm{s}}^{\mathrm{sr}}$ & & WA1805137, WA1983853, WA2229812 \\
\hline Turdus albicollis Vieillot, 1818 & 6,10 & fo & & \\
\hline \multicolumn{5}{|l|}{ Mimidae Bonaparte, 1853} \\
\hline Mimus saturninus (Lichtenstein, 1823) & $1,2,3,4,5,9,10$ & $\mathrm{ng}, \mathrm{sr}$ & & WA806208, WA1496259 \\
\hline Mimus triurus (Vieillot, 1818) + & 2 & sr & Am & WA1803618 \\
\hline \multicolumn{5}{|l|}{ Motacillidae Horsfield, 1821} \\
\hline Anthus lutescens Pucheran, 1855 & $1,2,4,5,6,7,8,9,10$ & $\mathrm{ng}, \mathrm{pa}$ & & WA1016940, WA1631470, WA1634952, WA1588845 \\
\hline Anthus hellmayri Hartert, $1909+$ & 2,4 & $\mathrm{ng}$ & & WA943270, WA988364 \\
\hline \multicolumn{5}{|l|}{ Passerellidae Cabanis \& Heine, 1850} \\
\hline Zonotrichia capensis (Statius Muller, 1776) & $1,2,3,4,5,6,7,8,9,10$ & ex, ng, pa, sr & & WA1808836, WA2229802 \\
\hline Ammodramus humeralis (Bosc, 1792) & $1,2,4,5,8,10$ & $\mathrm{ng}, \mathrm{pa}$ & & WA1803616, WA1633917 \\
\hline \multicolumn{5}{|l|}{ Parulidae Wetmore et al. 1947} \\
\hline Setophaga pitiayumi (Vieillot, 1817) & $1,2,3,4,5,6,7,8,9,10$ & fo, sr & & WA1593548 \\
\hline Geothlypis aequinoctialis (Gmelin, 1789) & $1,2,3,4,5,6,7,8,9,10$ & $\mathrm{ma}, \mathrm{sr}$ & & WA1805143 \\
\hline Basileuterus culicivorus (Deppe, 1830) & $1,4,6,7,8,10$ & $f_{0}, \mathrm{sr}$ & & WA1721314 \\
\hline Myiothlypis leucoblephara (Vieillot, 1817) + & 1 & fo & & WA2354735 \\
\hline \multicolumn{5}{|l|}{ Icteridae Vigors, 1825} \\
\hline Icterus pyrrhopterus (Vieillot, 1819) & $1,4,5,9$ & $\mathrm{fo}, \mathrm{sr}$ & & \\
\hline Amblyramphus holosericeus (Scopoli, 1786) & $1,2,3,4,6,7,8,9$ & ma & & WA890908, WA1749012, WA1590764, WA843600 \\
\hline Agelasticus thilius (Molina, 1782) & $1,2,3,4,6,7,8,9$ & ma & & WA1667251, WA1976483 \\
\hline Chrysomus ruficapillus (Vieillot, 1819) & $1,2,4,7,8,9,10$ & ma, om & & WA1740926 \\
\hline Xanthopsar flavus (Gmelin, 1788) + & 6 & pa & Vu-GL-BR, Cr-SC & WA2209033, WA2231008, WA2231010 \\
\hline Pseudoleistes guirahuro (Vieillot, 1819) & 1,2 & $\mathrm{ma}, \mathrm{ng}, \mathrm{pa}$ & & WA806226, WA1068021, WA806234, WA1987255 \\
\hline Pseudoleistes virescens (Vieillot, 1819) & $1,2,3,4,6,7,8,9$ & ma, ng, om & & WA1024447, WA1016342, WA1013612 \\
\hline Agelaioides badius (Vieillot, 1819) & $1,2,3,4,5,6,7,8,9,10$ & $\mathrm{ng}, \mathrm{pa}, \mathrm{sr}$ & & WA1803615, WA1976481, WA1586906 \\
\hline Molothrus bonariensis (Gmelin, 1789) & $1,2,3,4,5,6,7,8,9,10$ & ex, ng, sr ua & & WA1587905 \\
\hline Sturnella superciliaris (Bonaparte, 1850) & $1,2,4,5,6,7,8,9,10$ & ma, ng, pa & & WA1588850 \\
\hline \multicolumn{5}{|l|}{ Thraupidae Cabanis, 1847} \\
\hline Coereba flaveola (Linnaeus, 1758) & $1,4,5,6,7,9$ & $\mathrm{fo}_{0} \mathrm{sr}$ & & \\
\hline Tachyphonus coronatus (Vieillot, 1822) & $1,2,4,6,8,9,10$ & fo, sr & Atl & \\
\hline Coryphospingus cucullatus (Statius Muller, 1776) + & $1,4,5,8$ & ex, sr & & WA1803639, WA1497304, WA2228888 \\
\hline Dacnis cayana (Linnaeus, 1766) & 1,9 & $\mathrm{fo}_{\mathrm{s}} \mathrm{sr}$ & & WA1452839, WA1557863 \\
\hline Tangara sayaca (Linnaeus, 1766) & $1,2,3,4,5,6,7,8,9,10$ & fo, sr, ua & & WA1805135, WA1497302, WA2231005 \\
\hline Tangara preciosa (Cabanis, 1850) + & 8 & sr & & WA2576966 \\
\hline Paroaria coronata (Miller, 1776) + & 9 & pa & & WA1631411 \\
\hline Pipraeidea melanonota (Vieillot, 1819) + & 7 & fo & & WA1739502 \\
\hline Tersina viridis (Illiger, 1811) + & 2 & sr & & WA2378961 \\
\hline Donacospiza albifrons (Vieillot, 1817) & $3,4,5,8,9$ & ma & & WA1024446, WA1215401, WA1497303, WA988366 \\
\hline Sicalis flaveola (Linnaeus, 1766) & $1,2,3,4,5,6,7,8,9,10$ & ng, pa, ua & & WA1804437 \\
\hline Sicalis luteola (Sparrman, 1789) & $1,2,5,10$ & ma, ng, pa & & WA1976490, WA1178202 \\
\hline Emberizoides ypiranganus Ihering \& Ihering, 1907 & $1,2,4,5,9$ & ma, ng & & WA1082948, WA1082948, WA1068009 \\
\hline Embernagra platensis (Gmelin, 1789) & $1,2,3,4,5,6,7,7,8,9$ & $\mathrm{ma}, \mathrm{ng}$ & & WA1082945, WA806233, WA890868, WA1591749 \\
\hline Volatinia jacarina (Linnaeus, 1766) & $1,2,4,5,10$ & pa & & \\
\hline Sporophila caerulescens (Vieillot, 1823) & $2,3,4,5,6$ & $\mathrm{sr}$ & $\mathrm{Tm}$ & \\
\hline \multicolumn{5}{|l|}{ Cardinalidae Ridgway, 1901} \\
\hline Habia rubica (Vieillot, 1817) & 6 & fo & Atl & WA2045000 \\
\hline \multicolumn{5}{|l|}{ Fringillidae Leach, 1820} \\
\hline Spinus magellanicus (Vieillot, 1805) & $1,2,4,10$ & sr & & WA2354839 \\
\hline Euphonia chlorotica (Linnaeus, 1766) & 1,8 & fo, sr & & WA1739479, WA1016917 \\
\hline Euphonia violacea (Linnaeus, 1758) & 2,6 & $f_{0}$ & & WA1178079 \\
\hline Euphonia pectoralis (Latham, 1801) & 10 & fo & Atl & \\
\hline \multicolumn{5}{|l|}{ Estrildidae Bonaparte, 1850} \\
\hline Estrilda astrild (Linnaeus, 1758) & $1,2,4,9,10$ & ua & & WA1987253 \\
\hline \multicolumn{5}{|l|}{ Passeridae Rafinesque, 1815} \\
\hline Passer domesticus (Linnaeus, 1758) & $1,2,3,4,5,6,7,8,9,10$ & pa, ua & & WA1987270 \\
\hline
\end{tabular}




\section{Migratory species}

Overall, 38 species ( $17 \%$ of the total) are migrants (Table 2). Nearctic migrants are two shearwaters (Procellaridae), eight sandpipers (Scolopacidae), three plovers (Charadriidae), one tern (Sternidae), one jaeger (Stercorariidae), one cuckoo (Cuculidae), one falcon (Falconidae) and one swallow (Hirundinidae) species. Austral migrants are two albatrosses (Diomedeidae), one petrel (Procellaridae), two plovers, one penguin (Spheniscidae), one gull (Lariidae) and one mockingbird (Mimidae) species. Neotropical migrants are seven flycatchers (Tyrannidae), three swallows, one vireo
(Vireonidae) and one seedeater (Thraupidae) species (Table 2).

\section{Species of conservation concern}

\section{Spheniscus magellanicus (Forster, 1781)} (GL - NT; BR - LC; SC - LC)

On June $15^{\text {th }} 2013,65$ dead and three alive individuals (WA1804272) were found on the beach in Praia

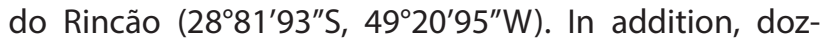
ens of individuals were found regularly every winter
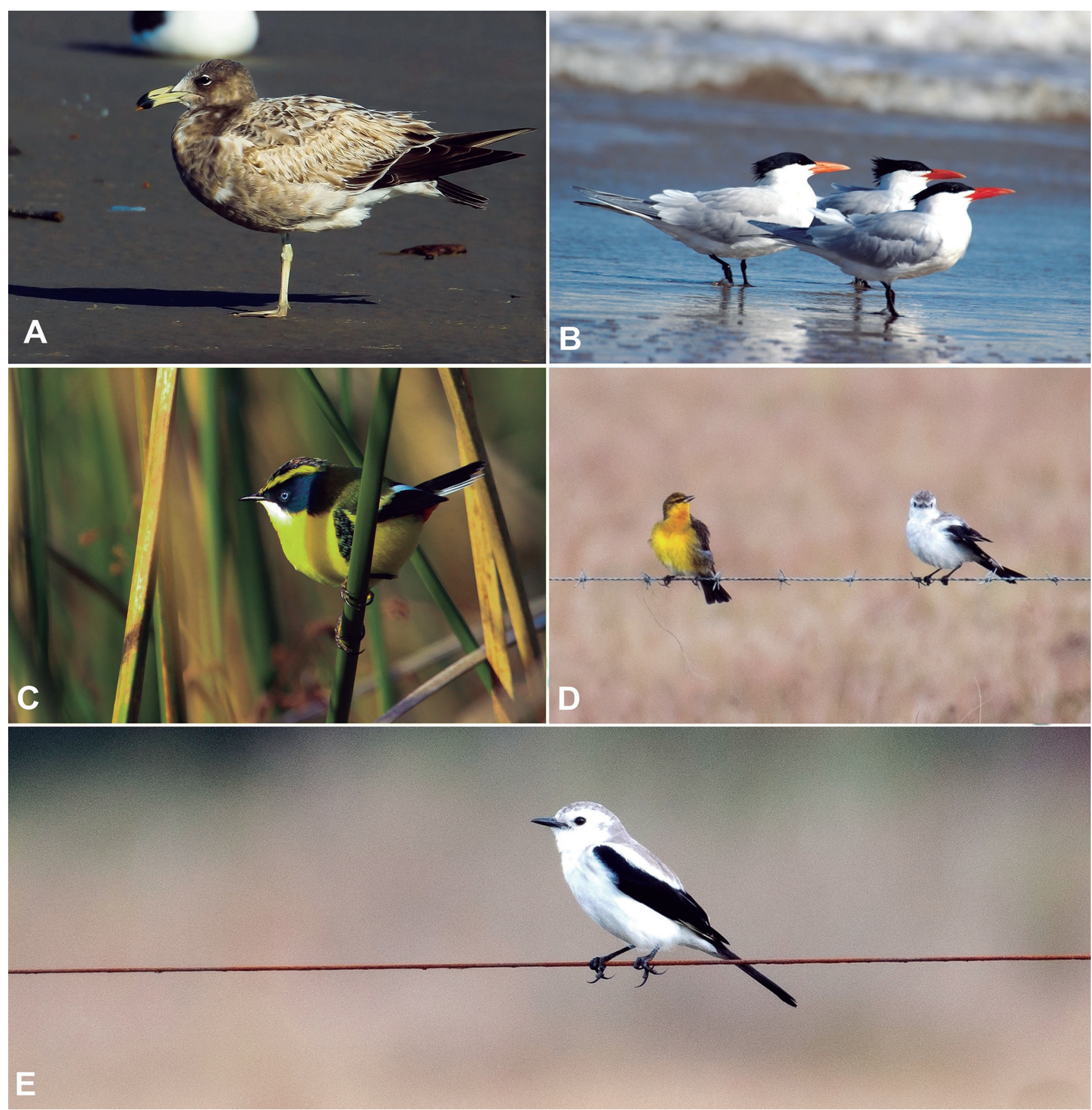

Figure 3. Records of conservation concern birds in the southern coast of Santa Catarina state, Brazil. (A) Larus atlanticus, Balneário Rincão; (B) Thalasseus maximus, Balneário Rincão; (C) Tachuris rubrigastra, Balneário Gaivota; (D) Xanthopsar flavus (yellow bird) and Xolmis dominicanus (black-and-white bird), Balneário Gaivota; (E) Xolmis dominicanus, Balneário Gaivota. 
(from the 2,000s up to moment) on the entire southern coast of Santa Catarina during beach monitoring programs made by the 'Museu de Zoologia Prof. Morgana Cirimbelli Gaidzinski' of the 'Universidade do Extremo Sul Catarinense' (R. Freitas, pers. comm.).

\section{Larus atlanticus Olrog, 1958 (GL - VU; BR - LC; SC - LC)}

On June $29^{\text {th }} 2014$, a juvenile was photographed (Fig. 3A; WA 1372730) and five other adults were observed in a mixed flock with Larus dominicanus (Lichtenstein, 1823) on the beach in Praia do Rincão. On August $28^{\text {th }}$ 2016, again an adult individual was recorded in Praia do Rincão (WA2257972).

\section{Gallinago undulata (Boddaert, 1783) (SC - VU)}

On $13^{\text {th }}$ January 2018, we heard for five times calls of two individuals and once barely observed them in a natural grassland used for livestock raising near the mouth of Urussanga River ( $\left.28^{\circ} 48^{\prime} 47.60^{\prime \prime} \mathrm{S}, 49^{\circ} 12^{\prime} 34.23^{\prime \prime} \mathrm{W}\right)$.

\section{Calidris canutus (Linnaeus, 1758) \\ (GL - NT; BR - CR; SC - LC)}

Several records during summer and autumn were obtained on the beach in Praia do Rincão: in 2013, it was recorded on April 21 ${ }^{\text {st }}$ (53 individuals; WA940368), October $6^{\text {th }}$ (one individual) and November $2^{\text {nd }}$ (four individuals); in 2014, it was recorded on March $30^{\text {th }}$ (70 individuals) and April 18 ${ }^{\text {th }}, 2014$ (158 individuals; WA1576078). In addition, on April 14 $4^{\text {th }}$ 2014, some small flocks were recorded near Araranguá river mouth (WA936332).

\section{Thalasseus maximus (Boddaert, 1783) (GL - LC; BR - EN; SC - VU)}

Several records during autumn, winter and spring were obtained on the beach in Praia do Rincão: in 2013, it was recorded on May $19^{\text {th }}$ (10 individuals), June $13^{\text {th }}$ (nine individuals) and June $15^{\text {th }}$ (four individuals; WA988660), July $13^{\text {th }}$ (nine individuals) and October $3^{\text {rd }}$ (eight individuals; Fig. 3B and WA1622675); in 2014, it was recorded on May $24^{\text {th }}$ (two individuals), June $29^{\text {th }}$ (two individuals) and June $26^{\text {th }}, 2014$ (10 individuals). In addition, some scattered flocks of three up to four individuals were recorded near Araranguá river mouth on April 21 ${ }^{\text {st }} 2013$ (WA960303).

\section{Sterna hirundinacea Lesson, 1831 (GL - LC; BR - VU; SC - LC)}

Several records were obtained during the winter, autumn and spring on the beach in Praia do Rincão: on October 14 2012 (two individuals; WA807753). In 2013, it was recorded on June $13^{\text {th }}$ (135 individuals), August $31^{\text {st }}$ (150 individuals), September $14^{\text {th }}$ (50 individuals), October $6^{\text {th }}$ (four individuals). In 2014, it was recorded on May $24^{\text {th }}$ (10 individuals), June $29^{\text {th }}$ (two individuals), July $26^{\text {th }}$ (650 individuals; WA2079160), August $24^{\text {th }}$ (80 indi- viduals) and September $21^{\text {st }}$ (four individuals). On April $21^{\text {st }} 2014$, a small flock was observed near Araranguá river mouth (WA1320064).

\section{Myrmotherula unicolor Ménétriès, 1835 (GL - NT; BR - LC; SC - LC)}

On March $6^{\text {th }} 2016$, a pair was observed and voice-recorded (WA2045047) in a patch of lowland forest north of Lagoa do Caverá (2900'49.02"S, 49³2'25.41"W).

\section{Geositta cunicularia (Vieillot, 1816) (GL - LC; BR - LC; SC - VU)}

This species was recorded year-round on the frontal dunes of Praia do Rincão. In 2013, it was recorded on March $17^{\text {th }}$ (three individuals), April $21^{\text {st }}$ (three individuals; WA942792), May 19th (one individual), September $14^{\text {th }}$ (one individual) and December $21^{\text {st }}$ (six individuals). In 2014, it was recorded on February $15^{\text {th }}$ (one individual), March $29^{\text {th }}$ (one individual), June $29^{\text {th }}$ (three individuals), July $26^{\text {th }}$ (one individual), August $24^{\text {th }}$ (one individual), October $19^{\text {th }}$ (one individual; WA1583389), November $22^{\text {nd }}$ (one individual) and December $13^{\text {th }}$ (two individuals). In 2015, it was recorded on January $16^{\text {th }}$ (one individual). In 2016, it was recorded on January $9^{\text {th }}$ (one individual) and $15^{\text {th }}$ (two individuals) and August $28^{\text {th }}$ (two individuals; WA2257977). In 2017, it was recorded on January $5^{\text {th }}$ (four individuals) and $6^{\text {th }}$ (two individuals; WA2433937) and February $2^{\text {nd }}$ (one individual).

\section{Phylloscartes kronei Willis \& Oniki, 1992 (GL - VU; BR - LC; SC - LC)}

On March $7^{\text {th }} 2015$, a pair was voice-recorded (WA1631446) and photographed (WA1634968) in Morro dos Macacos (29¹4'39.21"S, 4943'47.14"W). On December $31^{\text {st }} 2015$, one individual was heard near Lagoa da Urussanga Velha (28 $\left.46^{\prime} 42.42^{\prime \prime} S, 49^{\circ} 12^{\prime} 30.36^{\prime \prime} \mathrm{W}\right)$. On March $6^{\text {th }} 2016$, seven individuals were observed and some of them photographed (WA2046315) and voice-recorded (WA2045020) north of Lagoa do Caverá $\left(29^{\circ} 00^{\prime} 49.02^{\prime \prime} \mathrm{S}, 49^{\circ} 32^{\prime} 25.41^{\prime \prime} \mathrm{W}\right)$. On July $23^{\text {rd }} 2016$, four individuals were recorded (WA2231007) in the surroundings of Lagoa do Caverá (2905'21.46"S, 49³5'10.08"W). This species was also recorded near Lagoa da Urussanga Velha $\left(28^{\circ} 47^{\prime} 36.53^{\prime \prime} S, 49^{\circ} 12^{\prime} 04.28^{\prime \prime} \mathrm{W}\right)$ on November $5^{\text {th }}$ 2016 (four individuals), January $4^{\text {th }}$ (two individuals) and February 11 th 2017 (five individuals). All records made in patches of lowland forest, except one in an arbustive restinga (Lagoa da Urussanga Velha).

\section{Tachuris rubrigastra (Vieillot, 1817) (GL - LC; BR - LC; SC - VU)}

An individual was photographed (WA1739508) in Rio Novo $\left(29^{\circ} 09^{\prime} 50.80^{\prime \prime} S, 49^{\circ} 39^{\prime} 14.44^{\prime \prime} \mathrm{W}\right)$ on June $27^{\text {th }} 2015$. New records in this same site were made on July $5^{\text {th }} 2015$ (seven individuals; Fig. 3C) and on July 23 2016 (four individuals; WA2228844). All records were in marshes dominated by Schoenoplectus californicus. 


\section{Xolmis dominicanus (Vieillot, 1823) \\ (GL - VU; BR - VU; SC - EN)}

On July $16^{\text {th }} 2016$, five individuals were observed in the southwest of Lagoa do Caverá $\left(29^{\circ} 05^{\prime} 21.81^{\prime \prime} S\right.$, 49³3'43.17"W; WA2208102). On July 23 ${ }^{\text {rd }} 2016,15$ individuals along with six Xanthopsar flavus (Gmelin, 1788) were photographed in this same locality (Fig. 3D, Fig. 3E and WA2209035; WA2210218; WA2231009). Observations were made in overgrazed sandy grassland with sparse clumps of Baccharis trimera (Less.) DC and Pinus plantations.

\section{Xanthopsar flavus (Gmelin, 1788) (GL - VU; BR - VU; SC - CR)}

On July $23^{\text {rd }} 2016$, six individuals of Xanthopsar flavus along with 15 Xolmis dominicanus were photographed (Fig. 3D and WA2231008; WA2231010) in the same locality described above (i.e., 2905'21.81"S, 49³3'43.17"W).

\section{Distributional novelties}

\section{Calidris pusilla (Linnaeus, 1766)}

On March 29th 2018 , we observed an adult individual (WA2927732) in Praia do Rincão within a mixed flock of nearly 500 individuals of Calidris alba (Pallas, 1764).

\section{Aramides ypecaha (Vieillot, 1819)}

On September $25^{\text {th }}$, December $14^{\text {th }} 2014$, and August $23^{\text {rd }}$ 2015 we observed up to two individuals which were photographed (WA1496255) and voice-recorded (WA1495195) in Rio Novo $\left(29^{\circ} 09^{\prime} 50.89^{\prime \prime} \mathrm{S}, 4^{\circ} 39^{\prime} 16.20^{\prime \prime} \mathrm{W}\right)$ and Anita Garibaldi $\left(29^{\circ} 10^{\prime} 43.82^{\prime \prime} S, 49^{\circ} 40^{\prime} 21.04^{\prime \prime} W\right)$. On March $7^{\text {th }}$ 2015, one individual was photographed (WA1631483) in

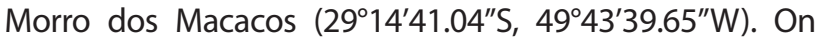
April 18 ${ }^{\text {th }}$ (WA1667252) and June 26 2015 (WA1739497), up to two individuals were photographed in Furnas $\left(29^{\circ} 07^{\prime} 34.17^{\prime \prime} \mathrm{S}, 49^{\circ} 39^{\prime} 52.55^{\prime \prime} \mathrm{W}\right)$. All records were in flooded grasslands and edges of marshes covered by Scirpus giganteus and Schoenoplectus californicus.

\section{Bubo virginianus (Gmelin, 1788)}

On July $3^{\text {rd }} 2015$, one individual was photographed (Fig. 4A) in Furnas $\left(29^{\circ} 07^{\prime} 35.46^{\prime \prime} \mathrm{S}, 4^{\circ} 39^{\prime} 56.40^{\prime \prime} \mathrm{W}\right)$. On August $23^{\text {rd }} 2015$, one individual was heard in Morro dos Macacos (29 $14^{\prime} 39.21^{\prime \prime}$ S, 49 $\left.43^{\circ} 47.14^{\prime \prime} \mathrm{W}\right)$. All records were in the edge of disturbed patches of lowland forest.

\section{Limnornis curvirostris Gould, 1839}

On June $3^{\text {rd }} 1991$, a specimen now deposited in the Museu Nacional do Rio de Janeiro, MN-37523 was collected by Marcos R. Bornschein and Marcos da Ré in "Lagoa do Sombrio, Furação, São João do Sul" (D. Figueira, in litt.);
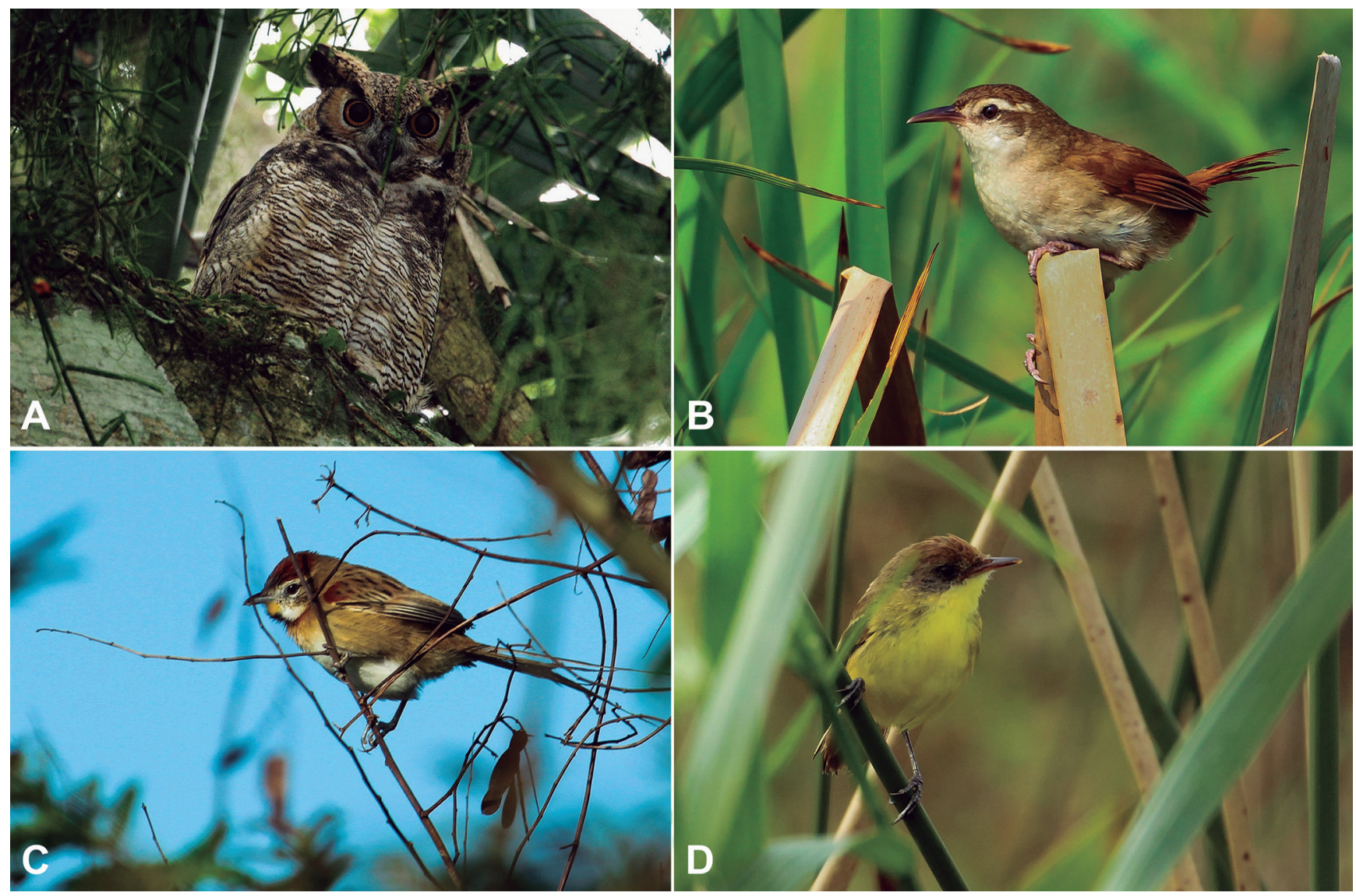

Figure 4. Birds considered novel distributional records for the southern coast of Santa Catarina state, Brazil. (A) Bubo virginianus, Sombrio; (B) Limnornis curvirostris, Balneário Gaivota; (C) Schoeniophylax phyrganophilus, Balneário Gaivota; (D) Pseudocolopteryx flaviventris, Balneário Gaivota. 
July $26^{\text {th }} 2003$, at least six individuals were observed in marshes on the eastearn border of 'Lagoa do Sombrio' (M.R. Bornschein \& G.N. Mauricio, pers. comm.); on April $11^{\text {th }} 2014$, a pair was photographed and voice-recorded (available at WikiAves, WA1296192 and WA1298515) in a marsh near 'Interpraias' highway, in Passo de Torres (29 $17^{\prime} 57.31^{\prime \prime}$ S, $\left.49^{\circ} 42^{\prime} 11.35^{\prime \prime} \mathrm{W}\right)$. On September $13^{\text {th }} 2014$ and July $5^{\text {th }} 2015$, up to two individuals were photographed (Fig. 4B; WA1748311) and voice-recorded in Anita Garibaldi (29 $\left.10^{\prime} 28.07^{\prime \prime S}, 49^{\circ} 40^{\prime} 22.01^{\prime \prime} \mathrm{W}\right)$. On December $14^{\text {th }} 2014$, April $18^{\text {th }}$ and May $7^{\text {th }} 2015$, up to four individuals were photographed (WA1667278) and voice-recorded (WA1668353) in Rio Novo (2909'48.22"S, 49³9'07.24"W). All records were in marshes dominated by Scirpus giganteus with small patches of Schoenoplectus californicus.

\section{Schoeniophylax phryganophilus (Vieillot, 1817)}

On July $5^{\text {th }} 2015$, one individual was photographed (Fig. 4C; WA1750012; WA1750038) and voice-recorded (WA1750015) in Anita Garibaldi (29 ${ }^{\circ} 10^{\prime} 38.98^{\prime \prime} S$, $\left.49^{\circ} 40^{\prime} 02.59^{\prime \prime} \mathrm{W}\right)$. On March $6^{\text {th }} 2016$, two individuals were observed and one of them photographed (WA2044970) and voice-recorded (WA2045008) in the southern border of Lagoa do Caverá, locality of Nova Guarita (2903'57.92"S, 49³5'10.02"W), Sombrio. All records were in pastures with sparse shrubs.

\section{Phacellodomus ferrugineigula (Pelzeln, 1858)}

On May $7^{\text {th }}$, June $27^{\text {th }}$ and August $23^{\text {rd }} 2015$, up to two individuals were photographed (WA1739509) and voice-recorded in Rio Novo (29 09'48.22"S, 49³9'07.24"W). On November $5^{\text {th }} 2016$ (four individuals; WA2354763; WA2354732), January $4^{\text {th }}$ (one individual) and February $11^{\text {th }} 2017$ (five individuals), the species was recorded along Urussanga river near Lagoa da Urussanga Velha ( $\left.28^{\circ} 47^{\prime} 31.41^{\prime \prime} \mathrm{S}, 49^{\circ} 12^{\prime} 00.44^{\prime \prime} \mathrm{W}\right)$. All records were in marshes dominated by Scirpus giganteus with sparse shrubs.

\section{Pseudocolopteryx flaviventris (d'Orbigny \& Lafresnaye, 1837)}

On $26^{\text {th }}$ July 2003 , voice-records of one individual were obtained in marshes in the eastern border of Lagoa do Sombrio (M.R. Bornschein \& G.N. Mauricio, pers. comm.). On October $13^{\text {th }} 2014$, two individuals were observed and one of them was photographed (Fig. 4D) in Anita Garibaldi (29 $\left.10^{\prime} 28.07^{\prime \prime S}, 49^{\circ} 40^{\prime} 22.01^{\prime \prime W}\right)$. On April $18^{\text {th }}$, June $27^{\text {th }}$, July $5^{\text {th }}$ and August $23^{\text {rd }} 2015$, up to two individuals were photographed (WA1667277; WA1744755; WA1748288) in Rio Novo (2909'48.22"S, 49³9'07.24"W).

\section{DISCUSSION}

\section{Bird diversity}

We present here a comprehensive update of the bird diversity and composition in the region of the coast- al lagoons in southern Santa Catarina. In total, the 250 species recorded here represents $36 \%$ of the avifauna of Santa Catarina state (700 species; Aves de Santa Catarina, 2016). Proper comparisons of bird diversity between ours and previous studies from surrounding areas are limited due to major differences in sampling and habitats considered. In coastal wetlands of Rio Grande do Sul, for example, the presence of multiple habitats, e.g., forests, marshes, and grasslands, has been shown to increase considerably the bird species richness (VizentinBugoni et al., 2015; Dias et al., 2016), which may be the case for coastal Santa Catarina as well, which encompass multiple habitats even in relatively short distances. Nevertheless, the total richness observed in our study can be considered intermediate in comparison to other coastal areas of southern Brazil: 120 species were recorded in Ibiraquera lagoon microbasin (Piacentini \& Campbell-Thompson, 2006), 227 species in Carijós Ecological Station (Vieira et al., 2014) and 228 species in Pirajubaé Extractive Marine Reserve (Vieira, 2016), all of them in Santa Catarina. Other inventories in the coast of Santa Catarina (e.g., Branco et al., 2004; Grose et al., 2013; Grose \& Cremer, 2015; Vieira et al., 2015) were restricted to few habitats (i.e., islands and beaches) and are too local for any comparison. For coastal wetland areas in Rio Grande do Sul, bird richness varies from 170 to 283 species (Vizentin-Bugoni et al., 2015).

Considering together ours and previous studies and records from online databases as WikiAves, specimens from museums and literature (Rosário-Bege \& Marterer, 1991; Rosário, 1996; Piacentini \& Campbell-Thompson, 2006; Piacentini et al., 2006), over 300 bird species have been reported to the region of coastal lagoons of southern Santa Catarina. However, further inventories will likely reveal new species for the region. The coast of southern Santa Catarina and Rio Grande do Sul are contiguous and similar in habitat composition (Burger, 1999; Menezes et al., 2015). Some species that occur in the coast of Rio Grande do Sul (Belton, 1994; Bencke et al., 2010) probably also occur in Santa Catarina, but were not found in this state yet due to low densities, inconspicuiness, migratory habits or because they use the region as stopover for only few days. This may be the case for several species, for example, Anas platalea Vieillot, 1816, Theristicus caerulescens (Vieillot, 1817), Coturnicops notatus (Gould, 1841), Cranioleuca sulphurifera (Burmeister, 1869), Calidris bairdii (Coues, 1861), Limnodromus griseus (Gmelin, 1789), Cinclodes fuscus (Vieillot, 1818) and Anthus furcatus d'Orbigny \& Lafresnaye, 1837.

\section{Distributional novelties}

A total of 68 species are novel records for the southern coast of the state in comparison to the previous broad-scale inventories (Rosário-Bege \& Marterer, 1991; Rosário, 1996). However, most of the novel species to the region are currently common and locally widely distributed with numerous recent records on online databases (e.g., WikiAves). Some of these species represent recent 
range expansions in Santa Catarina as Eupetomena macroura (Gmelin, 1788), Myiopsitta monachus (Boddaert, 1783), Zenaida auriculata (Des Murs, 1847) and Turdus leucomelas Vieillot, 1818 (e.g., Rosário, 1996; Straube et al., 2006) or are species difficult to detect due to inconspicuous behavior such as Botaurus pinnatus (Wagler, 1829), Ixobrychus involucris (Vieillot, 1823) and Bubo virginianus. We also recorded Schoeniophylax phryganophilus for the first time in Santa Catarina and presented the first records of Aramides ypecaha and Limnornis curvirostris in the field, which were to date known in the state only by single unpublished museum specimens. Larus atlanticus, Bubo virginianus and Phacellodomus ferrugineigula are known to the state by only one (Pacheco et al., 2009), four (Piacentini et al., 2006; Alves \& Pozenato, 2013; Just et al., 2015, 2016) and two (Minns et al., 2010; Just et al., 2015) previous records in literature, respectively. We also reported here the third and the first known populations of the globally threatened Xolmis dominicanus and Xanthopsar flavus, respectively, for the coast of Santa Catarina. As the nearest breeding populations of such species are known only for the coast of Rio Grande do Sul (Belton, 1994; Dias \& Maurício, 2002) and upland grasslands in Santa Catarina and Rio Grande do Sul (Repenning et al., 2010), our records likely correspond to wintering individuals.

\section{Conservation issues}

The mosaic of habitats including the coastal lagoons of southern Santa Catarina has been recognized as "an area of extreme importance for the conservation, sustainable use and benefit sharing of the Brazilian biodiversity" (MMA, 2007). Likewise, Schott \& Carbonell (1986) defined 'Lagoa do Sombrio' as an important area for the conservation of aquatic birds in the Neotropics. Despite these designations, there is currently only a few protected areas which cover small portions of natural habitats and are of sustanaible use, namely 'Área de Proteção Ambiental Baleia Franca' and 'Reserva Particular do Patrimônio Natural Capão Redondo' (14 ha). Additional protected areas have been created recently in the region of Praia do Rincão, namely 'Monumento Natural Morro dos Conventos' (280 ha), 'Área de Proteção Ambiental Costa de Araranguá' (3,213 ha) and 'Reserva Extrativista Rio Araranguá' extractive reserve (566 ha), but such reserves are not implemented yet and may be considered "paper parks" (Araranguá, 2016a, b, c). Importantly, the high diversity of Nearctic and Austral migrants and regionally threatened species found in the region of Praia do Rincão suggest that these protected areas may be especially important for the conservation of resident and migratory shorebirds.

Three threatened species, namely Larus atlanticus, Xolmis dominicanus and Xanthopsar flavus, were only scarsely recorded in the region and may be considered occasional visitors. Specifically, Larus atlanticus migrates northward after breeding in austral regions of South America, while the two other species are endemic to nat- ural grasslands of southeastern South America (Rosário, 1996). On the other hand, native habitats play an important role for the other nine threatened species in our study area. Lowland forests harbour high numbers of the vulnerable Phylloscartes kronei and other eight resident least concern species endemic to the Atlantic Forest. Mouths of Urussanga and Araranguá rivers and adjacent sandy beaches are important as wintering ground for a high number of long-distance migratory birds (Branco et al., 2004; this study), as breeding ground for some species as Sternula superciliaris (Vieillot, 1819) (Branco et al., 2004), Geositta cunicularia and Haematopus palliatus Temminck, 1820 (pers. obs.) and hold populations of regionally threatened birds, namely Thalasseus maximus, Calidris canutus, Sterna hirundinacea, and Geositta cunicularia. Marshy areas surrounding lagoons may be relevant in Santa Catarina to sustain high numbers of wetland-dependent species as the regionally threatened Tachuris rubrigastra and other least concern species as Pseudocolopteryx spp., Phleocryptes melanops (Vieillot, 1817), Limnornis curvirostris and Hymenops perspicillatus (Gmelin, 1789).

Notably, some bird species previously recorded in the region were not found in this study and the reasons may be diverse. Although some few species were likely undetected due to their cryptic habits, populations of other species may be decreasing or have gone locally extinct. This may be the case of species like: Rhea americana (Linnaeus, 1758), which is known for Santa Catarina only for a egg collected in 1934 in Sombrio (Rosário, 1996); Cygnus melancoryphus (Molina, 1782) and Netta peposaca (Vieillot, 1816) which were last recorded in the region almost 30 years ago (Sick et al., 1981; Rosário, 1996); Hydropsalis anomala (Gould, 1838) which was collected on 1991 in Sombrio (deposited in the Museu Nacional do Rio de Janeiro, MN-37515) (Rupp et al., 2007). There is also an intriguing undocumented report of the threatened Biatas nigropectus (Lafresnaye, 1850) for a lowland forest north of Lagoa do Caverá in 1988 (Rosário, 1996). Other records of this bamboo-dependent species in Santa Catarina are restricted to montane forests in the northeastern portion of the state (Rosário, 1996; distribution map available at WikiAves, www.wikiaves.com.br/ mapaRegistros papo-branco).

Additionally, the rate of records of some aquatic and wetland dependent species seems to have considerably decreased in the last three decades. For example, hundreds of individual of waterfowl species (Anatidae) were historically reported for the coastal lagoons of southern Santa Catarina (Rosário-Bege \& Marterer, 1991; Rosário, 1996), however, we recorded less than half of the Anatidae species mentioned for these studies and always in small flocks up to ten individuals. Other aquatic species of the families Podicipedidae (i.e., Podicephorus major (Boddaert, 1783), Podilymbus podiceps (Linnaeus, 1758) and Rollandia Rolland (Quoy \& Gaimard, 1824)) and Rallidae (Fulica armillata Vieillot, 1817) were also detected in low numbers and some other species were not even found (i.e., Fulica rufifrons Philippi \& Landbeck, 1861 and Porphyriops melanops (Vieillot, 1819)). Capture for pet trade and habitat loss may have extirpated the once 
frequent Sporophila collaris (Boddaert, 1783) from most suitable marshy areas in the region (Rosário, 1996), as it has few recent records (WikiAves, www.wikiaves.com.br/ mapaRegistros coleiro-do-brejo).

In regard to migratory birds, the lack of previous comprehensive studies on these groups has apparently hampered the inclusion of the southern coast of Santa Catarina in conservation initiatives (Bencke et al., 2006; Valente et al., 2011; CEMAVE/ICMBio, 2016). For instance, an attempt to identify important areas for Nearctic migratory birds in Brazil included adjacent Rio Grande do Sul and Santa Catarina states, but excluded the southern coast of the latter (Valente et al., 2011). Our records of 18 shorebirds species plus six other species from literature and WikiAves sum up 24 species of Nearctic migrants for southern Santa Catarina; additional species are Numenius hudsonicus Latham, 1790 (Silva, 2010), Calidris himantopus (Bonaparte, 1826) (Willrich et al., 2015), Calidris minutilla (Vieillot, 1819) (Leal, 2015), Calidris subruficollis (Vieillot, 1819) (Machado, 2012), Arenaria interpres (Linnaeus, 1758) (Bianco, 2009) and Phalaropus tricolor (Vieillot, 1819) (Santos, 2012). This high richness compared to other important stopover areas (Valente et al., 2011; CEMAVE/ICMBio, 2016) demonstrates the importance of southern Santa Catarina for several migratory species. Thus, we strongly recommend that future initiatives on migratory birds carefully consider the southern coast of Santa Catarina, especially sandy beaches from Laguna to Passo de Torres.

In summary, we demonstrated that the region of the coastal lagoons of southern Santa Catarina harbours high bird richness, including several threatened, endemic and long-distance migratory species. We believe this inventory presents crucial basic information useful to support further conservation strategies concerning this important coastal area in southern Brazil. The regional avifauna would be benefited by the implementation and proper management of the existing protected areas. Moreover, areas of high diversity of threatened and migratory birds such as Praia do Rincão and Lagoa do Sombrio, may be priority regions for the establishment of new protected areas and conservation efforts. We also encourage further surveys in such areas specially focused on assessing the abundances of endemic, threatened and migratory species. Further research aiming to investigate the consequences of anthropic activities on the avifauna is also necessary in order to understand the extent of such impacts, especially fragmentation of wetlands and grasslands, plantation of large stands of exotic trees, traffic on beaches and drainage of wetlands.

\section{ACKNOWLEDGEMENTS}

We thank to Antônio D'Ávila da Silva, Antônio Gonçalves Júnior, Jorge Manoel Rodrigues and Nelson Matias Rodrigues for permission to access their properties; Ana Paula Ribeiro, Bianca Maximo, Bento T.L. Junior, Caroline de C. Freitas, Daniela Bôlla, Diego D. Pavei, Marcos C. Pazetto, Sérgio Moreira, Alexandre Bianco,
Gilberto J. Botelho and Ricardo D. Colvero for assitance in some field surveys; A. Bianco, G.J. Botelho (Figure 2A, B, C and Figure 3D, E) and R.D. Colvero for documentation of some species and photographs; Daniel Figueira, Vitor de Q. Piacentini and Marcos R. Bornschein for providing data on the specimen of Limnornis curvirostris housed in MNRJ; Giovanni N. Mauricio and M.R. Bornschein for providing unpublished records; Carla S. Fontana (curator) and her staff for providing access and support at the bird collection in Pontifícia Universidade Católica do Rio Grande do Sul; anonymous reviewers, editor Luis Fábio Silveira, Bianca P. Vieira, Jeferson Vizentin-Bugoni and Rafael A. Dias for valuable suggestions on a first draft of the manuscript; Jeferson Vizentin-Bugoni and Daniel G. Kovar for linguistic revision; Fundação de Amparo à Pesquisa e Inovação do Estado de Santa Catarina (FAPESC) for funding part of this research. PIBIC/UNESC and PIC/170 for the grants conceded along the research.

\section{REFERENCES}

Alves, R.V.Z. \& Pozenato, G.S. 2013. Registro de Bubo virginianus em Floresta Ombrófila Mista no estado de Santa Catarina, sul do Brasil. Atualidades Ornitológicas, 170: 24-25.

Amorim, J.F. \& Piacentini, V.Q. 2006. Novos registros de aves raras em Santa Catarina, Sul do Brasil, incluindo os primeiros registros documentados de algumas espécies para o Estado. Revista Brasileira de Ornitologia, 14: 145-149.

Amorim, J.F. \& Piacentini, V.Q. 2007. Novas áreas de ocorrência de três Passeriformes no sul do Brasil. Lundiana, 8: 69-73.

Araranguá. 2016a. Dispõe sobre a criação da unidade de conservação da natureza municipal Monumento Natural Morro dos Conventos, e dá outras providências. Decreto № 7829. Araranguá, Secretária da Administração Municipal, 27 de dezembro de 2016.

Araranguá. 2016b. Dispõe sobre a criação da unidade de conservação da natureza municipal Área de Proteção Ambiental (APA) da Costa de Araranguá, e dá outras providências. Decreto № 7828. Araranguá, Secretária da Administração Municipal, 27 de dezembro de 2016.

Araranguá. 2016c. Dispõe sobre a criação da unidade de conservação da natureza municipal Reserva Extrativista do Rio Araranguá, e dá outras providências. Decreto № 7830. Araranguá, Secretária da Administração Municipal, 27 de dezembro de 2016.

Aves de Santa Catarina. 2016. Banco de dados da avifauna catarinense. Available at: http://avesdesantacatarina.com.br. Access in: 03/02/2016.

Azevedo, M.A.G. \& Ghizoni-Jr., I.R. 2005. Novos registros de aves para o Estado de Santa Catarina, sul do Brasil. Atualidades Ornitológicas, 126: 9-12.

Belton, W. 1994. Aves do Rio Grande do Sul: distribuição e biologia. São Leopoldo, Editora Unisinos.

Bencke, G.A.; Burger, M.I.; Dotto, J.C.P.; Guadagnin, D.L.; Leite, T.O. \& Menegheti, J.0. 2007. Aves. In: Becker, F.G.; Ramos, R.A. \& Moura, L.A. (Orgs.). Biodiversidade RS. Regiões da Lagoa do Casamento e dos Butiazais de Tapes, Planície Costeira do Rio Grande do Sul. Brasília, Ministério do Meio Ambiente. p. 314-353.

Bencke, G.A.; Dias, R.A.; Bugoni, L.; Agne, C.E.; Fontana, C.S.; Maurício, G.N. \& Machado, D.B. 2010. Revisão e atualização da lista das aves do Rio Grande do Sul, Brasil. Iheringia Série Zoologia, 100: 519-556.

Bencke, G.A.; Maurício, G.N.; Develey, P.F. \& Goerck, J.M. (Orgs.). 2006. Áreas Prioritárias para a Conservação de aves no Brasil. Parte I - estados do domínio da Mata Atlântica. São Paulo, SAVE Brasil. 
Bianco, A. 2009. WA45604, Arenaria interpres (Linnaeus, 1758). Available at: www.wikiaves.com/45604. Access in: 29/04/2016.

Branco, J.0.; Machado, I.F. \& Bovendorp, M.S. 2004. Avifauna associada a ambientes de influência marítima no litoral de Santa Catarina, Brasil. Revista Brasileira de Zoologia, 21: 459-466.

Burger, M.I. 1999. Situação e ações prioritárias para a conservação de banhados eáreas úmidas da zona costeira. Available at: www.anp.gov.br/ brasilrounds/round8/round8/guias r8/perfuracao r8/\%C3\%81reas Priorit\%C3\%A1rias/Banhados.pdf. Access in: 15/03/2016.

Burger, M.I. \& Ramos, R.A. 2007. Áreas importantes para a conservação na planície costeira do Rio Grande do Sul. In: Becker, F.G.; Ramos, RA \& Moura, L.A. (Orgs.). Biodiversidade do Rio Grande do Sul: regiões da lagoa do Casamento e dos Butiazais de Tapes, planície costeira do Rio Grande do Sul. Brasília, Ministério do Meio Ambiente. 58p.

Carlos, C.J. \& Voisin, J.F. 2013. The type locality of the South American Tern, Sterna hirundinacea (Aves: Charadriiformes: Sternidae), with designation of a lectotype. Zoologia, 30: 356-358.

Centro Nacional de Pesquisa e Conservação das Aves Silvestres, Instituto Chico Mendes de Conservação da Biodiversidade (CEMAVE/ICMBio). 2016. Relatório anual de rotas eáreas de concentração de aves migratórias no Brasil. Available at: www.icmbio.gov.br/portal/images/stories/DCOM Miolo Rotas Migrat\%C3\%B3rias 2016 final.pdf. Access in: 25/04/2016.

Chesser, R.T. 1994. Migration in South America: an overview of the austral system. Bird Conservation International, 4: 91-107.

Conselho Estadual do Meio Ambiente (CONSEMA). 2011. Lista oficial de espécies da fauna ameaçadas de extinção no Estado de Santa Catarina e dá outras providências - Resolução CONSEMA № 002 de 06 de dezembro de 2011. Florianópolis, Diário Oficial, CONSEMA. 19p.

Dias, R.A. \& Maurício, G.N. 2002. Natural history notes and conservation of a Saffron-cowled Blackbird Xanthopsar flavus population in the southern coastal plain of Rio Grande do Sul, Brazil. Bird Conservation International, 12: 255-268.

Dias, R.A.; Maurício, G.N. \& Bugoni, L. 2016. Birds of the Patos Lagoon Estuary and adjacent coastal waters, southern Brazil: species assemblages and conservation implications. Marine Biology Research, 1-13.

Esteves, F.A.; Caliman, A.; Santangelo, J.M.; Guariento, R.D.; Farjalla, V.F. \& Bozelli, R.L. 2008. Neotropical coastal lagoons: an appraisal of their biodiversity, functioning, threats and conservation management. Brazilian Journal of Biology, 68: 967-981.

Falkenberg, D.B. 1999. Aspectos da flora e da vegetação secundária da restinga de Santa Catarina, Sul do Brasil. Insula: Revista de Botânica, 28: $1-30$.

Ghizoni-Jr., I.R. \& Azevedo, M.A.G. 2010. Registro de algumas aves raras ou com distribuição pouco conhecida em Santa Catarina, sul do Brasil, e relatos de três novas espécies para o estado. Atualidades Ornitológicas, 154: 33-46.

Giannini, P.C.F.; Sawakuchi, A.0. \& Martinho, C.T. 2007. Eolian depositional episodes controlled by Late Quaternary relative sea level changes on Imbituba-Laguna coast (southern Brazil). Marine Geology, 237: 143-168.

Grose, A.V. \& Cremer, M.J. 2015. Aves migratórias no litoral norte de Santa Catarina, Brasil. Ornithologia, 8: 22-32.

Grose, A.V.; Hillebrant, C.C. \& Cremer, M.J. 2013. Diversidade e abundância sazonal da avifauna em duas planícies de maré no estuário da baía da Babitonga, norte de Santa Catarina. Iheringia Série Zoologia, 103: 6-11.

Guadagnin, D.L. \& Laidner, C. 1999. Diagnóstico da Situação e Ações Prioritárias para a Conservação da Zona Costeira da Região Sul - Rio Grande do Sul e Santa Catarina. Porto Alegre, Pronabio-Funbio.

Guadagnin, D.L. \& Maltchik, L. 2007. Habitat and landscape factors associated with neotropical waterbird occurrence and richness in wetland fragments. Biodiversity \& Conservation, 16: 1231-1244.
Guadagnin, D.L.; Maltchik, L. \& Fonseca, C.R. 2009. Species-area relationship of Neotropical waterbird assemblages in remnant wetlands: looking at the mechanisms. Diversity and Distribution, 15: 319-327.

Guadagnin, D.L.; Peter, Â.S.; Perello, L.F.C. \& Maltchik, L. 2005. Spatial and temporal patterns of waterbird assemblages in fragmented wetlands of southern Brazil. Waterbirds, 28: 261-272.

Harrison, N.M.; Whitehouse, M.J. \& Madureira, L.A.S.P. 2013. Observations of the under-described avifauna of the Mostardas Peninsula, Rio Grande do Sul, Brazil. Check List, 9: 391-399.

Hellmayr, C.E. 1936. Catalogue of birds of the Americas and the adjacent islands: part IX, Tersinidae - Thraupidae. Field Museum of Natural History Zoological Series, 13: 1-480.

International Union for Conservation of Nature (IUCN). 2015. The IUCN Red List of threatened species. Version 2015.2. International Union for Conservation of Nature. Available at: www.iucnredlist.org. Access in: 01/01/2015.

Just, J.P.G.; Romagna, R.S.; Rosoni, J.R.R. \& Zocche, J.J. 2015. Avifauna na região dos contrafortes da Serra Geral, Mata Atlântica do sul de Santa Catarina, Brasil. Atualidades Ornitológicas, 187: 33-54.

Just, J.P.G.; Viana, I.R. \& Zocche, J.J. 2016. Liophis miliaris (Watersnake). Predation. Herpetological Review, 47: 147-147.

Klein, A.S.; Citadini-Zanette, V. \& Santos, R. 2007. Florística e estrutura comunitária de restinga herbácea no município de Araranguá, Santa Catarina. Biotemas, 20: 15-26.

Leal, S.J. 2015. WA1913047, Calidris minutilla (Vieillot, 1819). Available at: www.wikiaves.com/1913047. Access in: 01/05/2016.

Machado, 0.J. 2012. WA783526, Calidris subruficollis (Vieillot, 1819). Available at: www.wikiaves.com/783526. Access in: 01/05/2016.

Mähler-Jr., J.K.F.; Kindel, A. \& Kindel, E.A.I. 1996. Lista comentada das espécies de aves da Estação Ecológica do Taim, Rio Grande do Sul, Brasil. Acta Biologica Leopoldensia, 18: 69-113.

Menezes, L.S.; Müller, S.C. \& Overbeck, G.E. 2015. Floristic and structural patterns in South Brazilian coastal grasslands. Anais da Academia Brasileira de Ciências, 87: 2081-2090.

Minns, J.; Buzzetti, D.; Albano, C.; Grosset, A.; Whittaker, A. \& Parrini, R. 2010. Aves do Brasil, vozes e fotografias. Floresta Atlântica, Cerrado, Caatinga, Pantanal, Campos Sulinos e Costa. Volume 1 (DVD-ROM). Vinhedo, Avis Brasilis Editora.

Ministério do Meio Ambiente (MMA). 2002. Biodiversidade Brasileira: avaliação e identificação de ações prioritárias para a conservação, utilização sustentável e repartição dos benefícios da biodiversidade Brasileira. Brasília, Ministério do Meio Ambiente.

Ministério do Meio Ambiente (MMA). 2007. Áreas Prioritárias para a Conservação, uso sustentável e repartição dos recursos da biodiversidade Brasileira: Atualização - Portaria MMA № 9 de 23 de janeiro de 2007. Brasília, Ministério do Meio Ambiente.

Ministério do Meio Ambiente (MMA). 2014. Lista nacional oficial de espécies da fauna ameaçadas de extinção - Portaria MMA № 444 de 17 de dezembro de 2014. Brasília, Diário Oficial da União 245: 121-126. Available at: www.mma.gov.br/biodiversidade/especiesameacadas-de-extincao. Access in: 24/07/2015.

Nascimento, I.L.S. 1995. As aves do Parque Nacional da Lagoa do Peixe. Brasília, IBAMA.

Pacheco, J.F.; Branco, J.0. \& Piacentini, V.Q. 2009. Olrog's Gull Larus atlanticus in Santa Catarina, Brazil: northernmost occurrence and first state record. Cotinga, 31: 149-150.

Piacentini, V.Q. \& Campbell-Thompson, E.R. 2006. Lista comentada da avifauna da microbacia hidrográfica da Lagoa de Ibiraquera, Imbituba, SC. Biotemas, 19: 55-65.

Piacentini, V.Q.; Aleixo, A.; Agne, C.E.; Maurício, G.N.; Pacheco, J.F.; Bravo, G.A.; Brito, G.R.R.; Naka, L.N.; Olmos, F.; Posso, S.; Silveira, L.F.; Betini, G.S.; 
Carrano, E.; Franz, I.; Lees, A.C.; Lima, L.M.; Pioli, D.; Schunck, F.; Amaral, F.R.; Bencke, G.A.; Cohn-Haft, M.; Figueiredo, L.F.A.; Straube, F.C. \& Cesari, E. 2015. Annotated checklist of the birds of Brazil by the Brazilian Ornithological Records Committee/Lista comentada das aves do Brasil pelo Comitê Brasileiro de Registros Ornitológicos. Revista Brasileira de Ornitologia, 23: 91-298.

Piacentini, V.Q.; Ghizoni-Jr., I.R.; Azevedo, M.A.G.; Carrano, E.; Borchardt-Jr., C.A.; Amorim, J.F. \& Grose, A.V. 2009. Ocorrência, expansão e distribuição do maçarico-de-cara-pelada Phimosus infuscatus (Lichtenstein, 1823) (Ciconiiformes: Threskiornithidae) no Estado de Santa Catarina, sul do Brasil. Revista Brasileira de Ornitologia, 17: 107-112.

Piacentini, V.Q.; Ghizoni-Jr., I.R.; Azevedo, M.A. \& Kirwan, G.M. 2006. Sobre a distribuição de aves em Santa Catarina, Brasil, Parte I: registros relevantes para 0 Estado ou inéditos para a Ilha de Santa Catarina. Cotinga, 26: 25-31.

Pinto, 0.M.0. 1938. Catalogo das aves do Brasil e lista dos exemplares que as representam no Museu Paulista. Revista do Museu Paulista, 22: 1-556.

Repenning, M.; Rovedder, C.E. \& Fontana, C.S. 2010. Distribuição e biologia de aves nos campos de altitude do sul do Planalto Meridional Brasileiro. Revista Brasileira de Ornitologia, 18: 283-306.

Rosário, L.A. 1996. As aves em Santa Catarina: distribuição geográfica e meio ambiente. Florianópolis, FATMA.

Rosário-Bege, L.A.\& Marterer, B.T.P. 1991. Conservação da avifauna na região sul do Estado de Santa Catarina-Brasil. FATMA, Florianópolis.

Rupp, A.E.; Brandt, C.S.; Fink, D.; Thom-Silva, G.; Laps, R.R. \& Zimmermann, C.E. 2007. Registros de Caprimulgiformes e a primeira ocorrência de Caprimulgus sericocaudatus (bacurau-rabode-seda) no Estado de Santa Catarina, Brasil. Revista Brasileira de Ornitologia, 15: 605-608.

Santos, E.C. 2012. WA564689, Phalaropus tricolor (Vieillot, 1819). Available at: www.wikiaves.com/564689. Access in: 01/05/2016.

Schott, D.A. \& Carbonell, M. 1986. A directory of Neotropical wetlands. Cambridge, IUCN.

Sick, H.; Rosário, L.A. \& Azevedo, T.R. 1981. Aves do Estado de Santa Catarina: lista sistemática baseada em bibliografia, material de museu e observação de campo. Sellówia Série Zoologia, 1: 1-52.

Silva, R.R.V. 2003. Contribuição à avifauna observada na praia do Mar Grosso em Laguna, SC. Biotemas, 16: 181-187.
Silva, T.D. 2010. WA133414, Numenius hudsonicus Latham, 1790. Available at: www.wikiaves.com/133414. Access in: 01/05/2016.

Somenzari, M.; Amaral, P.P.; Cueto, V.R.; Guaraldo, A.C.; Jahn, A.E.; Lima, D.M.; Lima, P.C.; Lugarini, C.; Machado, C.G.; Martinez, J.; Nascimento, J.L.X.; Pacheco, J.F.; Paludo, D.; Prestes, N.P.; Serafini, P.P.; Silveira, L.F.; Sousa, A.E.B.A.; Sousa, N.A.; Souza, M.A.; Telino-Júnior, W.R. \& Whitney, B.M. 2018. An overview of migratory birds in Brazil. Papéis Avulsos de Zoologia, 58: e20185803.

Straube, F.C. 2010. Reavaliação dos registros históricos de araras para o estado de Santa Catarina. Atualidades Ornitológicas, 157: 75-84.

Straube, F.C.; Urben-Filho, A. \& Piacentini, V.Q. 2006. 0 beija-flor tesoura Eupetomena macroura (Gmelin, 1788) e sua ampliação de distribuição pelo sul do Brasil. Atualidades Ornitológicas, 132: 49-51.

Teixeira, M.B.; Coura-Neto, A.B.; Pastore, U. \& Rangel Filho, A.L.R. 1986. Vegetação: as regiões fitoecológicas, sua natureza e seus recursos econômicos estudo fitogeográfico. In: IBGE. Levantamento de recursos naturais. Rio de Janeiro, IBGE. p. 541-620. (Volume 33).

Tomazelli, L.J. \& Villwock, J.A. 2005. Mapeamento geológico de planícies costeiras: 0 exemplo da costa do Rio Grande do Sul. Gravel, 3: 109-115.

Valente, R.M.; Silva, J.M.C.; Straube, F.C. \& Nascimento, J.L.X. 2011. Conservação de aves migratórias Neárticas no Brasil. Belém, Conservação Internacional.

Vieira, B.P. 2016. Composição de aves da reserva marinha extrativista do Pirajubaé, Santa Catarina, e implicações para sua gestão e conservação. Atualidades Ornitológicas, 189: 33-44.

Vieira, B.P.; Dias, D.; Piacentini, V.Q.; Correia, E.C. \& Serafini, P.P. 2014. Birds of Estação Ecológica de Carijós, southern Brazil. Check List, 10: 1110-1122.

Vieira, B.P.; Dias, D.; Rocha, H.J.F. \& Serafini, P.P. 2015. Birds of the Arvoredo Marine Biological Reserve, southern Brazil. Check List, 11: 1532.

Vizentin-Bugoni, J.; Jacobs, F.; Coimbra, M.A.A. \& Dias, R.A. 2015. Birds of the Reserva Biológica do Mato Grande and surroundings, Rio Grande do Sul, Brazil. Check List, 11: 1641.

Willrich, G.; Deconto, L.R. \& Vallejos, M.A.V. 2015. Calidris himantopus (Scolopacidae) no estado de Santa Catarina, sul do Brasil. Atualidades Ornitológicas, 188: 26-27. 


\section{APPENDIX}

Bird species undetected in our ten study sites during field work but with previous records from literature (i.e., Rosário, 1996; Branco et al., 2004; Rupp et al., 2007) or WikiAves (author and catalogue number available at the website - WA). Localities: 2 = Praia do Rincão, 4 = Foz do Rio Araranguá, 6 = Lagoa do Caverá, $7=$ Furnas, $8=$ Rio Novo, $9=$ Anita Garibaldi. Status: $\mathrm{Atl}=$ species endemic to the Atlantic Forest, $\mathrm{En}=$ endangered, $\mathrm{Vu}=$ vulnerable, $\mathrm{Nt}=$ near threatened, $\mathrm{GL}=\mathrm{global}$ level, $\mathrm{SC}=$ state level, $\mathrm{Nm}=$ Nearctic migrant, $\mathrm{Tm}=$ Neotropical migrant.

\begin{tabular}{|c|c|c|c|c|}
\hline Taxon & Localities & Date of record & Source & Status \\
\hline Rhea americana (Linnaeus, 1758) & 7 & 1934 & Rosário (1996) & \\
\hline Cygnus melancoryphus (Molina, 1782) & 2,8 & 10 Nov. 1987, 28 Apr. 1980 & Rosário (1996) & \\
\hline Netta peposaca (Vieillot, 1816) & $7,8,9$ & 3 Sep. and 18 Dec. 1982, 25 Aug. 1992 & Rosário (1996) & \\
\hline Dendrocygna bicolor (Vieillot, 1816) & 6,8 & 19 Dec. 1982, 25 Aug. 1992 & Rosário (1996) & \\
\hline Anas bahamensis Linnaeus, 1758 & 6,8 & 18 Jul. 1988, 25 Aug. 1992 & Rosário (1996) & \\
\hline Fulica rufifrons Philippi \& Landbeck, 1861 & $7,8,9$ & 28 and 29 Apr. 1980, 15 Jul. 1988, 25 Aug. 1992 & Rosário (1996) & \\
\hline Porphyriops melanops (Vieillot, 1819) & $2,6,8$ & 15 and 18 Jul. and 14 Aug. 1988 & Rosário (1996) & \\
\hline Limosa haemastica (Linnaeus, 1758) & 4 & 21 Out. 2015 & E. Morisso (WA1887303) & $\mathrm{Nm}$ \\
\hline Actitis macularius (Linnaeus, 1766) & 4 & 2002 or 2003 & Branco et al. (2004) & $\mathrm{Nm}$ \\
\hline Phalaropus tricolor (Vieillot, 1819) & 4 & 10 Jan. 2011 & I. Ghizoni-Jr. (WA370198) & $\mathrm{Nm}$ \\
\hline Chroicocephalus cirrocephalus (Lichtenstein, 1823) & 4 & 1Sep. 2009 & I. Ghizoni-Jr. (WA599792) & \\
\hline Hydropsalis anomala (Gould, 1838) & 8 & 3 June 1991 & Rupp et al. (2007) & Nt-GL, En-SC \\
\hline Hylocharis chrysura (Shaw, 1812) & 4,8 & 26 Apr. 2014; 30 Jul. 2016 & J.C. Campos (WA1320844); B. Junior (WA2301999) & \\
\hline Florisuga fusca (Vieillot, 1817) & 4 & 5 Feb. 2012 & J.C. Campos (WA803233) & \\
\hline Falco peregrinus Tunstall, 1771 & 4 & 14 Apr. 2015 & R. Cardoso (WA1663881) & $\mathrm{Nm}$ \\
\hline Biatas nigropectus (Lafresnaye, 1850) & 6 & 18 Jul. 1988 & Rosário (1996) & Atl, Vu-GL-SC \\
\hline Pyrocephalus rubinus (Boddaert, 1783) & 4 & 21 Sep. 2014, 21 0ct. 2016 & E. Morisso (WA1460203, WA2351796) & $\operatorname{Tm}$ \\
\hline Turdus flavipes Vieillot, 1818 & & & E. Morisso (WA1899049) & \\
\hline Tangara palmarum (Wied, 1821) & 4 & 17 Sep. 2014 & J.C. Campos (WA1454507) & \\
\hline Hemithraupis guira (Linnaeus, 1766) & 4 & 15 Nov. 2015 & E. Morisso (WA1914459) & \\
\hline Hemithraupis ruficapilla (Vieillot, 1818) & 4 & $210 c t .2015$ & E. Morisso (WA1887286) & Atl \\
\hline Pipraeidea bonariensis (Gmelin, 1789) & 4 & 25 Dec. 2011 & I. Teixeira (WA1112011) & \\
\hline Sporophila collaris (Boddaert, 1783) & 8 & 18 Dec. 1982 and 25 Aug. 1992; 6 May 2015 & Rosário (1996); L. Deconto (WA1691009) & \\
\hline
\end{tabular}

